\title{
Physical and chemical properties of selected beef muscles infused with a phosphate and lactate blend
}

\author{
L.C. Hoffman ${ }^{1 \#}$, A. Vermaak ${ }^{1,2}$ \& N. Muller ${ }^{2}$ \\ ${ }^{1}$ Department of Animal Sciences, Stellenbosch University, Private Bag X1, Matieland, Stellenbosch, 7602, South \\ Africa \\ ${ }^{2}$ University of Stellenbosch, Department of Food Science, Private Bag X1, Matieland 7602, South Africa
}

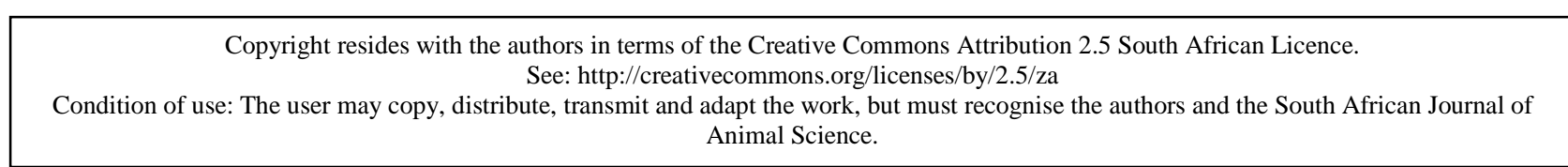

\begin{abstract}
The consumer demands a beef product of consistent and acceptable tenderness. The infusion of beef muscles with a blend containing sodium and potassium salts, various phosphates and lactates has the potential to improve the current status of low meat consumption and inconsistent tenderness of fresh beef products in South Africa. In the present investigation, the biceps femoris (BF, silverside), rectus femoris muscle (RF), semitendinosus muscle (ST, eye of the silverside), supraspinatus muscle (SS, scotch fillet) and longissimus et lumborum muscles from the left side of beef carcasses were infused, $3 \mathrm{~d}$ post mortem, with a blend consisting of various sodium and potassium salts, di- and triphosphates and lactates, while the corresponding muscles from the right side were untreated and served as the control. The changes in beef quality over a 19-d period and the initial proximate and mineral composition of the muscles were also determined. The general findings suggest that an increase in tenderness concurrent with an acceptable beef colour resulted from the infusion with this blend. The chemical composition of the treated muscles was not negatively affected by the infusion and the mineral content of the treated muscles was increased, accordingly.
\end{abstract}

Keywords: Alkaline infusion, $\mathrm{pH}$, water-binding capacity, instrumental tenderness, beef colour, proximate composition, mineral composition

\# Corresponding author: lch@sun.ac.za

\section{Introduction}

Attending to the consumer demand for fresh meat products of consistent quality is of great importance in achieving success in the meat industry and increasing beef consumption. A major weakness in the modern beef industry is the variability of beef quality, and in particular tenderness (Morgan et al., 1991b; Smith et al., 1992). Several studies on meat acceptability have indicated that consumers consider tenderness the most important attribute (Whipple et al., 1990) and surely the most desirable when meat is consumed, whether at home or in a restaurant (Huffman et al., 1996). Other important qualities that consumers consider when buying meat are freshness, juiciness and the nutrients provided by the product (Boleman et al., 1995; Grunert, 1997).

Meat tenderness varies among species, animals within the same species, and among muscles (Polidori et al., 2000).

Beef colour is another important beef quality trait that has shown variation during retail display (Got et al., 1999). Even though colour is considered a poor guide to eating quality, consumers base their purchase decisions on colour display (Young et al., 1999).

Over the years, several techniques and processes have been researched and applied in search of a solution to the problem of meat-quality variation and in particular tenderness. These include electrical stimulation (Dransfield et al., 1992; Simmons et al., 2008), carcass suspension (Sørheim \& Hildrum, 2002) and muscle stretching (Toohey et al., 2012a; b), natural ageing (Lawrie, 1998), blade tenderisation (BenitoDelgado et al., 1994; Pietrasik \& Shand, 2011), marination (Scanga et al., 2000), injection (McGee et al., 
2003) and explosion (Solomon et al., 1997). Meat-enhancing agents such as phosphates and salts have been investigated and their successes have been documented (Kerth et al., 1995; Morris et al., 1997; Holmer et al., 2009). Enhancing the flavour, tenderness and consumer acceptance of retail beef products and the ability to produce value-added and water-added beef products creates a growing market opportunity in the beef industry (Scanga et al., 2000). Several injection/infusion solutions that consist mainly of calcium and sodium salts have been developed. Examples include sodium lactate, known for its flavour-enhancing and shelf-lifeextension properties (Duxbury, 1988; Maca et al., 1999), and sodium phosphate, used to increase protein solubility and water-binding ability (Hellendoorn, 1962; Trout \& Schmidt, 1984). A solution of calcium chloride $\left(\mathrm{CaCl}_{2}\right)$ infused into meat has been demonstrated to be successful in enhancing and accelerating post-mortem tenderisation (Koohmaraie et al., 1988; 1989; 1990; Koohmaraie \& Shackelford, 1991; Morgan et al., 1991a; Wheeler et al., 1991).

Phosphates are typically a component of enhancement solutions in the modern beef industry, because of their ability to increase the functionality of meat products, particularly via water binding (Hamm, 1970; Trout \& Schmidt, 1983). Water retention in fresh muscles is based on a buffered (with phosphates) water solution with a $\mathrm{pH}$ that is more alkaline and further away from the isoelectric point of the meat. This action increases the water-holding capacity of the meat (Mandigo, 2002).

Phosphates and sodium chloride $(\mathrm{NaCl})$ increase functionality via protein swelling (Paterson et al., 1988), ionic strength and $\mathrm{pH}$ (Trout \& Schmidt, 1984). This increased functionality leads to increased water retention (Trout \& Schmidt, 1983) and improved tenderness and juiciness (Prestat et al., 2002). Therefore, the inclusion of salt and phosphate improves the yield and palatability characteristics and affects the colour and shelf-life. Contradictory colour results have been reported with the use of a phosphate and $\mathrm{NaCl}$ blend. Meat colour is either improved (Lee et al., 1998) or diminished (Chen \& Trout, 1991) with the infusion of such a blend.

The post-mortem storage (ageing) of beef at chill temperatures has been the practice for many years, and remains an important procedure for producing tender meat in the modern meat industry (Koohmaraie et al., 1988). It is known that different muscles from one carcass react differently to post-mortem storage (Koohmaraie et al., 1988; Rhee et al., 2004). A possible solution is the infusion of a blend containing salts, phosphates and lactates. Our laboratory have shown that this technology is suitable for decreasing the time required for ageing meat, even when applied to old and tough muscles (Hoffman, 2006). However, muscles respond to the same extent when infused (Molina et al., 2005). The study by Molina et al. found that brine injection reduced the percentage cook loss in seven of the eight beef shoulder muscles evaluated. However, it had no significant effect on Warner-Bratzler shear force (WBSF) values and sensory tenderness ratings of five and four muscles, respectively. It is postulated that the increase in tenderness is the result of physical damage caused by the injecting needles as well as the improved water-binding capacity owing to the infused phosphate and lactate salts. The improved water-binding capacity also causes a diluting effect on the protein responsible for meat texture.

The present study investigates a commercially available basting (Freddy Hirsch Tenderbite \# 802539) consisting of sodium and potassium salts, various phosphates and lactates. This brine was used to infuse biceps femoris (BF, silverside), rectus femoris (RF), semitendinosus (ST, eye of the silverside), supraspinatus (SS, scotch fillet) and longissimus et lumborum (LL, striploin) beef muscles. Previous research (Hoffman, 2006) has indicated that this specific blend increases the tenderness of meat significantly. However, the effect of the blend on beef qualities, with post-mortem ageing, has not yet been determined. Therefore, the first aim of this study is to ascertain the effect of a phosphate and lactate blend on the physical ( $\mathrm{pH}$, water-binding capacity, beef colour and shear force) and chemical properties (proximate and mineral composition) of selected beef muscles. A secondary aim is to establish whether the blend has any significant effect on the physical properties over a given time.

\section{Materials and Methods}

Beef carcasses representing South African beef breeds (Brahman $\times$ Simmentaler cross; $n=3$, average mass $=301 \mathrm{~kg}$ and Charolais $\times$ Hereford cross; $\mathrm{n}=3$, average mass $=298 \mathrm{~kg}$ ) finished in a feedlot, were sourced from a commercial abattoir in Paarl, Western Cape, South Africa. At the abattoir, the animals were slaughtered, dressed and processed according to standard South African techniques and conditions. No electrical stimulation was applied to the carcasses. The animals were selected to represent steers from a typical commercial scenario, representative of the South African market. The carcasses were classified as A2 
according to the South African classification system (Government Notice No R. 1748, 26 June 1992). An A2 animal is a young animal of the A age group (no permanent incisors) with a fat code of 2, representing a lean fat cover (1 - $3 \mathrm{~mm}$ thick subcutaneous fat depth measured between the 10th and 11th ribs, $50 \mathrm{~mm}$ from the midline of the cold unquartered carcass). The whole intact carcasses were chilled at $c a .2{ }^{\circ} \mathrm{C}$ for $24 \mathrm{~h}$ in a cooling chamber before being weighed and quartered at the abattoir (Day 1). Twenty-four hours (Day 2) post mortem (pm) the beef quarters were moved into a mobile cooling unit (set at $4{ }^{\circ} \mathrm{C}$ ) and transported to the Meat Science Laboratory at Stellenbosch University, where the carcasses were stored in the cooling facility at $4{ }^{\circ} \mathrm{C}$. On the same day (Day 2; $24 \mathrm{~h} \mathrm{pm}$ ) the left- and right-side B, RF, ST, SS and LL muscles were removed from the carcasses, trimmed of all visible subcutaneous fat and superficial collagen, weighed, labelled, vacuum packed and stored in a cooler at $\mathrm{ca} .4^{\circ} \mathrm{C}$ until further processing.

On Day 3 (48 h pm) all the muscles were transported to the Freddy Hirsch Processing Plant, where they were removed from their packaging, demembraned, reweighed to determine the pre-infusion weight and the $\mathrm{pH}$ was measured. Muscles from the right side of the carcass were left untreated and stored in a cooler at $2{ }^{\circ} \mathrm{C}$ to be used as the control. The muscles from the left side were infused with a salt mixture containing sodium and potassium di- and triphosphates, sodium lactate and sodium chloride (Freddy Hirsch Tenderbite; PO Box 2554, Cape Town, 8000) at a pressure of 2.4 bar at 30 strokes per min on a Rühle Curing Centre IR56 (Rühle GmbH, D·79865, Grafenhausen, Germany) to give a calculated pumped gain of 15\% with a retention of $12 \%$. The basting mixture gave a calculated chemical composition of $75.8 \%$ water, $5.21 \% \mathrm{Na}^{+}$, $2.53 \% \mathrm{~K}^{+}, 3.45 \% \mathrm{P}_{2} \mathrm{O}_{5}$ and $12.4 \%$ lactate. The treated muscle samples were weighed immediately after infusion and after a resting (equilibration) period of $2 \mathrm{~h}$ to calculate the retained pumped gain. After $2 \mathrm{~h}$ the 10 muscles from both sides were divided into six equal portions by cutting across the length of the muscles. Each portion was randomly allocated to each time point.

The six time intervals reflected six successive post-mortem periods of measurements: days $4,7,10$, 13, 16 and 19. Meat cuts were cut cross-sectionally to the muscle fibre to determine $\mathrm{pH}$, purge loss, drip loss, cooking loss, colour and shear force of fresh beef muscle $\left(4^{\circ} \mathrm{C}\right)$. The same muscle segments of the left and right were compared experimentally. After the division, the muscles (sub-samples) were weighed, labelled, vacuum packed, stored in crates, transported back to the Meat Science Laboratory, and stored in the cooler at $4{ }^{\circ} \mathrm{C}$ until collected for analysis on the pre-assigned day.

On the sampling date the samples allocated to the time interval were removed from the cooler for analyses. On analysing the physical characteristics of the muscle, the sample surfaces were dried with absorbent paper and reweighed to calculate purge loss (exudate collected in the vacuum bag). Meat slices of approximately $1.5 \mathrm{~cm}$ thick were cut cross-sectionally to the muscle fibre to determine the instrumental colour (CIE Lab) of the raw (after a blooming period of $30 \mathrm{~min}$ ) (Wulf \& Wise, 1999) and cooked muscles, drip loss, cooking loss and instrumental tenderness of the cooked muscles.

On sampling day 4, the remainder of the samples were homogenised, vacuum packed and stored at $-18^{\circ} \mathrm{C}$ until proximate chemical and mineral analyses could be conducted.

The physical characteristics determined from the deboned muscles consisted of the $\mathrm{pH}$ before and after infusion, the pumped gain and purge loss. The data collected from the sub-samples over the 19-d period were $\mathrm{pH}$, purge loss, drip loss, cooking loss, raw and cooked colour and instrumental tenderness (WBSF). The $\mathrm{pH}$ measurements were conducted with a penetrating glass electrode on a hand-held Crison $\mathrm{pH} / \mathrm{mV}$-507 meter with an automatic temperature compensator.

The left-side muscles were weighed before and immediately after infusion to calculate the pumped gain, as well as $2 \mathrm{~h}$ after infusion (stored at $2{ }^{\circ} \mathrm{C}$ ) to calculate the retained pumped gain. The purge losses of the undivided infused muscles were calculated from the pumped gain measurements.

Purge loss, drip loss and cooking loss, and colour were determined by the methods described by Honikel (1998). $\mathrm{L}^{*}, \mathrm{a}^{*}$ and $\mathrm{b}^{*}$ colour measurements were taken using a Colour-guide $45^{\circ} / 0^{\circ}$ colorimeter (Catalog No 6805; BYK-Gardner, USA). These ordinates were used to calculate hue angle and chroma (Honikel, 1998) using the following equations (CIE, 1978):

$$
\text { Chroma: } C^{*}=\sqrt{\left(a^{*}\right)^{2}+\left(b^{*}\right)^{2}} \quad \text { Hue angle: } h_{a b}=\tan ^{-1}\left\{\frac{b^{*}}{a^{*}}\right\}
$$

After measuring the cooking loss, the same samples were stored overnight in a refrigerator $\left(4^{\circ} \mathrm{C}\right)$ and used for instrumental determination of tenderness the next morning. The shear force values of the cooked meat samples were obtained with a Warner-Bratzler shear (WBS) attachment (Voisey, 1976), fitted to an 
Instron Universal Testing Machine (Model 4444). Tenderness was measured as the maximum force (Newton) required to shear a $1.27 \mathrm{~cm}$ diameter cylindrical core of cooked meat (perpendicular to the grain) at a crosshead speed of $200 \mathrm{~mm} / \mathrm{min}$.

The total percentage of moisture, protein, fat and ash of the raw beef muscle samples was determined according to AOAC methods (AOAC, 2002). The total lipid content was determined by extracting the fat with a $1: 2$ mixture of chloroform and methanol (Lee et al., 1996). The moisture content was analysed by drying a $2.5 \mathrm{~g}$ sample at $100{ }^{\circ} \mathrm{C}$ for a period of $24 \mathrm{~h}$ (method 934.01, AOAC, 2002) and ashing by cremating the samples at $500{ }^{\circ} \mathrm{C}$ for $6 \mathrm{~h}$. The protein content was determined by the Dumas combustion method (Method 968.06, AOAC, 2002) on the defatted samples using a FP528 nitrogen analyser.

The mineral composition of the meat was determined after ashing defatted meat samples. These samples (1 - 3 g) were air-dried and ground to pass through a $0.5 \mathrm{~mm}$ to $1.0 \mathrm{~mm}$ sieve. After this the samples were ashed overnight in a muffle furnace at $550{ }^{\circ} \mathrm{C}$. A $6 \mathrm{~N}$ hydrochloric acid (HCI) solution was prepared by diluting $500 \mathrm{~mL}$ of a $36 \%(\mathrm{~m} / \mathrm{m}) \mathrm{HCI}$ solution to 1 litre. After ashing, $5 \mathrm{~mL}$ of a $6 \mathrm{M}$ HCI was added to dissolve the cooled sample. After cooling, a $5 \mathrm{~mL} 6 \mathrm{~N}$ nitric acid $\left(\mathrm{HNO}_{3}\right)$ solution was added to the samples. The $6 \mathrm{~N} \mathrm{HNO}_{3}$ solution was prepared by diluting $429 \mathrm{~mL}$ of a $65 \%(\mathrm{~m} / \mathrm{m})$ solution to $1 \mathrm{~L}$. After adding this solution, the samples were heated in a water bath and removed after boiling point was reached. The solution was subsequently filtered through filter paper into a $100 \mathrm{~mL}$ volumetric flask and diluted to volume with deionised water (Giron, 1973).

The concentrations of calcium $(\mathrm{Ca})$, copper $(\mathrm{Cu})$, iron $(\mathrm{Fe})$, potassium $(\mathrm{K})$, magnesium $(\mathrm{Mg})$, sodium $(\mathrm{Na})$, phosphorus $(\mathrm{P})$, lead $(\mathrm{Pb})$ and zinc $(\mathrm{Zn})$ of the digestates were determined by using the inductively coupled plasma spectrometry (ICP) detection method (Method No AgriLASA 6.1.1) (Handbook of Feed \& Plant Analysis, Volume 2).

The experimental design for the deboned whole muscles was a randomised complete block design with 10 treatment combinations replicated in six blocks (animals/carcasses). The treatment design was a $2 \times 5$ factorial with the factors, two treatments (control and infused) and five muscles (BF, RF, ST, SS and $\mathrm{LL}$ ). The $\mathrm{pH}$ and pumped data were measured before infusion and after $2 \mathrm{~h}$ equilibration (resting period) and differences were calculated. All these data were subjected to an analysis of variance using SAS Statistical Software Version 9.1 (SAS, 2003). The Shapiro-Wilk test was performed to test for non-normality (Shapiro \& Wilk, 1965). Student's t-least significant difference (t-LSD) was calculated at the 5\% confidence level to compare treatment means of significant source effects (Ott, 1998).

A further statistical analysis was conducted on the muscles to test the effect of the infusion solution with a storage period of $19 \mathrm{~d}$ on the physical parameters $(\mathrm{pH}$, purge loss, drip loss, cooking loss, shear force, and raw and cooked colour). The treatment design was a $2 \times 6$ factorial experiment replicated in six blocks (animals/carcasses). The factors were two treatments (control \& infused), and six time periods (days 4, 7, 10, $13,16,19)$ determined for the five individual muscles (BF, RF, ST, SS and LL). Analyses of variance were performed for all of these variables using SAS Statistical Software Version 9.1 (SAS, 2003). The ShapiroWilk test was performed to test for non-normality (Shapiro \& Wilk, 1965). Student's t-LSD was calculated at the $5 \%$ confidence level to compare treatment means of significant source effects (Ott, 1998).

Another statistical analysis was conducted on the muscles to test for the effect of the infusion on the chemical parameters (proximate and mineral composition). The design was a $2 \times 5$ factorial experiment replicated in six blocks (animals/carcasses) with factors two treatments (control \& infused) and five muscles (BF, RF, ST, SS and LL). Factorial analysis of variance were performed on the chemical constituents measured, using SAS Statistical Software Version 9.1 (SAS, 2003). The Shapiro-Wilk test was performed to test for non-normality (Shapiro \& Wilk, 1965). Student's t-LSD was calculated at the 5\% confidence level to compare treatment means of significant source effects (Ott, 1998).

\section{Results and Discussion}

For all the parameters tested, there were no interaction among the main effects and thus they are discussed in more detail. The results from the deboned muscles infused with the phosphate and lactate blend on Day 3 (pre- and post-infusion pH, pumped gain) are depicted in Table 1. In Table 2 the mean values for the physical meat quality parameters of $\mathrm{pH}$, water-binding properties and shear force resistance of the $\mathrm{BF}$, RF, ST, SS, and LL sub-samples are displayed. In Table 3 the data for the quality measurements of the muscles over the $19 \mathrm{~d}$ were pooled and the muscles means are compared within and between treatments. 
Several studies have shown that in order to improve the WHC of processed meat, the $\mathrm{pH}$ should be increased to a desired point (Young et al., 2005). This is achieved by adding an alkalinising agent to the meat product, such as alkaline polyphosphates (Shults et al., 1972; Puolanne et al., 2001). This agent aids the saltinduced solubilisation of myosin and augments water binding by increasing the $\mathrm{pH}$ (Young et al., 2005).

From Table 1 it is clear that the samples of both pre-infusion treatments were reasonably similar in initial $\mathrm{pH}$ values on the third day post mortem. Before infusion of the blend (Day 3; $72 \mathrm{~h} \mathrm{pm}$ ), the $\mathrm{pH}$ of the control samples ranged from $5.45 \pm 0.043$ (LL) to $5.52 \pm 0.055$ (RF), and the $\mathrm{pH}$ of the samples earmarked for infusion ranged from $5.38 \pm 0.035$ (LL) to $5.53 \pm 0.064$ (SS). After infusion the $\mathrm{pH}$ of the infused muscles increased substantially to a pH range of $5.55 \pm 0.189$ for LL to $5.77 \pm 0.232$ for SS (Table 1). This increase in $\mathrm{pH}$ was expected and is supported by many studies, in which the effect of an alkaline solution containing polyphosphates on muscle $\mathrm{pH}$ is researched (Baublits et al., 2005). The pH difference of the control and infused muscles (Table 1$)$ illustrated differences before infusion $(P \leq 0.05)$ for the LL muscle, whereas after infusion there were no differences in $\mathrm{pH}$ between pre- and post-infusion muscles $(P>0.05)$, illustrating that infusion decreased $\mathrm{pH}$ differences between muscles.

Bendall (1967) reported that phosphates increased the volume of uncooked muscles. This statement is supported by the present investigation, with an increase in muscle volume after infusion. The percentage fluid retained (pumped gain) directly after the muscles were infused ranged from $18.05 \pm 2.299$ (BF) to $22.93 \pm 3.312$ (SS) at $0 \mathrm{~h}$ and then decreased to $13.73 \pm 2.916$ (LL) to $17.59 \pm 3.928$ (RF) after a $2 \mathrm{~h}$ stabilisation period (Table 1). Previous studies reported similar pumped gain values (Hoffman, 2006).

The results pertaining to the specific change of the $\mathrm{pH}$ in each muscle over time are given in Table 2 . The $\mathrm{pH}$ of the infused samples differed $(P \leq 0.05)$ from that of the control over the $19 \mathrm{~d}$, indicating that the phosphate blend increased the muscle $\mathrm{pH}$ of the infused samples substantially. The $\mathrm{pH}$ of the samples also changed $(P \leq 0.05)$ over the 19-d period. The general trend in both the control and infused muscles was that of an initial increase from Day 4 to 13 , and then the $\mathrm{pH}$ started to decrease $(P \leq 0.05)$ from Day 13 to 16 . Several authors reported that the alkalinity of the muscles, and thus the $\mathrm{pH}$, is increased when muscles were treated with a blend containing phosphates (Boles \& Shand, 2001; Baublits et al., 2005) and with the infusion of sodium lactate (Maca et al., 1999). All the muscles showed a decrease in $\mathrm{pH}$ towards the end of the shelf-life study - the reason for this phenomenon is not clear although it is speculated that it could be linked to bacterial growth - unfortunately this aspect was not evaluated.

The significant effect of the phosphate blend on the muscle $\mathrm{pH}$ illustrated in Table 2 should result in a significant effect on the water-binding abilities of the muscle (Honikel, 1987; Scanga et al., 2000; Baublits et al., 2005) and more specifically purge loss, drip loss and cooking loss (Briskey et al., 1960; Crouse et al., 1984). Several studies reported that steaks marinated in a solution of higher $\mathrm{pH}$ and strong buffering capacity have increased water-binding ability compared with steaks left untreated or marinated in solutions with a $\mathrm{pH}$ close to, or below, the isoelectric point of meat (Trout \& Schmidt, 1986; Boles \& Shand, 2001; McGee et al., 2003; Baublits et al., 2005).

In the present investigation the fluid-loss measurements consisted of the determination of purge loss (collected in vacuum bags over time), drip and cooking loss observed within the infused and control muscles. The control gives an indication of the normal fluid loss and of the water-holding capacity (WHC) of the meat under these circumstances, where fresh meat is stored in vacuum bags at a chill temperature. The WHC of muscles treated with a phosphate and lactate blend is known as the water-binding capacity (WBC) of the infused meat, which is the ability of the meat to bind added water (Boleman et al., 1995).

In the present investigation (Table 2$)$ there was no difference $(P>0.05)$ in purge loss between the infused and control muscles. Lawrence et al. (2003) found similar results, that is, a slightly higher, but not significant purge loss in muscles treated with a salt solution. The addition of salt to a solution increases the ionic strength of the solution, thereby increasing the number of hydrophilic protein interactions, which causes an increase in the binding of free water (Lawrence et al., 2003). In the present investigation the amount of drip loss was higher for the infused samples than for the control samples, with differences for BF, ST and SS $(P \leq 0.05)$ (Table 3$)$. Several other studies reported this effect, with a consistent increase in WHC associated with an increase in salt content (Hamm, 1960; Sherman, 1962; Wheeler et al., 1993; Lennon et al., 2006).

Several authors reported a significant reduction in cooking loss when treating muscle with a salt solution similar to that of the present study (Bouton et al., 1982; Sheard et al., 1999; Walsh et al., 2010). Most of the infused muscles in the current investigation (Table 2) did not have higher cooking loss values 
than the untreated muscles $(P>0.05)$. The relatively similar cooking loss values of the control and infused muscles indicate that infusion did not have a negative effect on cooking loss in this investigation. However, there were differences within some of the muscles over storage time. For example, the BF and RF control and infused muscles differed $(P \leq 0.05)$ from Day 4 to 13 , after which both treatments stabilised and showed similar cooking losses $(P>0.05)$. Generally, the cooking loss of the LL, ST and SS control and infused samples (Table 2) followed a similar pattern $(P>0.05)$. Other authors also reported results of infused muscles indicating numerically higher cooking loss, but similar to the untreated muscles $(P>0.05)$ (Baublits et al., 2006).

Table 3 illustrates the overall effect between treatments and between muscles for $\mathrm{pH}$ and waterbinding capacity. The $\mathrm{pH}$, purge, drip and cooking loss increased $(P \leq 0.05)$ with infusion in most of the muscles. The WBSF values of the various muscles measured over time are given in Table 2. A treatment effect $(P \leq 0.05)$ was achieved in the present study when a phosphate and salt solution was used to infuse the beef muscles, with reduced WBSF values obtained for all the infused samples on the designated days. This result illustrates that infusion has a substantial and positive effect on meat tenderness. Vote et al. (2000) report significant treatment differences between control and infused samples. Stites et al. (1989) found that when beef roasts were injected with a solution containing sodium tripolyphosphate and sodium chloride the WBSF values were significantly lowered when compared with those of the control samples. Authors such as McGee et al. (2003) have shown that the injection of a sodium lactate-phosphate-chloride brine in beef inside round roasts resulted in decreased instrumental tenderness.

The time effect showed that all the muscles illustrated differences in tenderness $(P \leq 0.05)$ over time (Table 2). Both the control and infused muscles showed a pattern of decreased shear force with time from Day 7 to 19. Therefore, over time a fair amount of conditioning (ageing and tenderisation) took place in both treatments. The initial shear force of some of the untreated and infused muscles was low on Day 4 and then increased to Day 7. No clear explanation could be found to support this result. Reports on the effect of ageing on tenderness are contradictory. Some studies reported no influence of ageing on WBSF, whereas others found a significant decrease in WBSF values throughout the ageing period, thus a significant improvement in tenderness over time (French et al., 2001; Maria et al., 2003).

Table 3 illustrates the overall effect between treatments and between muscles for WBSF values. The shear force decreased substantially $(P \leq 0.05)$ with infusion in all the muscles. This trend illustrates the positive effect of infusion on meat tenderness. Support muscles are reported to be more tender than locomotive muscles (Belew et al., 2003). However, with infusion this factor is not relevant, suggesting that the blend tenderised all the muscles to an acceptable level (Hoffman et al., 2008). In this investigation the infused muscles BF (38.90 N), RF (36.06 N) and LL (41.08 N) had significantly lower WBSF values than ST $(47.63 \mathrm{~N})$ and SS $(47.26 \mathrm{~N})$. The relatively high $\mathrm{pH}$ of the latter two samples could be ascribed to the initial high $\mathrm{pH}$ of the untreated samples. 
Table 1 Means $( \pm \text { s.d.) })^{\#}$ for infusion data on Day 3 of beef muscles infused with a phosphate and lactate blend

\begin{tabular}{|c|c|c|c|c|c|c|c|c|}
\hline \multirow{2}{*}{ Muscle } & \multicolumn{2}{|c|}{ Pre-infusion $\mathrm{pH}$} & \multirow{2}{*}{$\begin{array}{l}\mathrm{pH} \text { difference } \\
\left(\text { control }^{\mathrm{d}} \text { vs. }\right. \\
\left.\text { infused }^{\mathrm{e}}\right) \\
\end{array}$} & \multirow{2}{*}{$\begin{array}{c}\text { Post-infusion } \mathrm{pH}^{\mathrm{f}} \\
\text { Infused }\end{array}$} & \multirow{2}{*}{$\begin{array}{l}\text { pH difference } \\
\text { (pre }^{\mathrm{e}} \text { vs. post } \\
\text { infusion) } \\
\end{array}$} & \multirow{2}{*}{$\begin{array}{c}\text { Pumped gain (\%) } \\
0 \mathrm{~h}^{\mathrm{g}} \\
\text { Infused }\end{array}$} & \multirow{2}{*}{$\begin{array}{c}\begin{array}{c}\text { Pumped gain (\%) } \\
2 \mathrm{~h}^{\mathrm{h}}\end{array} \\
\text { Infused }\end{array}$} & \multirow{2}{*}{$\begin{array}{c}\begin{array}{c}\text { Pumped gain } \\
\text { difference }(\%)^{\mathrm{g}-\mathrm{h}}\end{array} \\
\text { Infused }\end{array}$} \\
\hline & Control $^{\mathrm{d}}$ & Infused $^{\mathrm{e}}$ & & & & & & \\
\hline $\mathrm{BF}$ & $5.45^{b} \pm 0.022$ & $5.42^{\mathrm{bc}} \pm 0.038$ & $-0.03^{\mathrm{ab}} \pm 0.027$ & $5.72^{\mathrm{a}} \pm 0.266$ & $0.31^{\mathrm{a}} \pm 0.286$ & $18.05^{\mathrm{b}} \pm 2.299$ & $14.81^{b} \pm 2.152$ & $3.24^{\mathrm{b}} \pm 1.824$ \\
\hline $\mathrm{RF}$ & $5.52^{\mathrm{a}} \pm 0.055$ & $5.47^{\mathrm{b}} \pm 0.045$ & $-0.06^{\mathrm{b}} \pm 0.031$ & $5.68^{\mathrm{a}} \pm 0.144$ & $0.21^{\mathrm{a}} \pm 0.164$ & $22.14^{\mathrm{a}} \pm 3.601$ & $17.59^{\mathrm{a}} \pm 3.928$ & $4.55^{\mathrm{b}} \pm 1.005$ \\
\hline ST & $5.45^{\mathrm{b}} \pm 0.034$ & $5.40^{\mathrm{C}} \pm 0.025$ & $-0.05^{\mathrm{ab}} \pm 0.050$ & $5.68^{\mathrm{a}} \pm 0.272$ & $0.28^{\mathrm{a}} \pm 0.264$ & $19.43^{\mathrm{ab}} \pm 4.881$ & $15.72^{\mathrm{ab}} \pm 4.797$ & $3.71^{b} \pm 1.894$ \\
\hline SS & $5.51^{\mathrm{a}} \pm 0.055$ & $5.53^{\mathrm{a}} \pm 0.064$ & $0.02^{\mathrm{a}} \pm 0.091$ & $5.77^{\mathrm{a}} \pm 0.232$ & $0.24^{\mathrm{a}} \pm 0.249$ & $22.93^{\mathrm{a}} \pm 3.312$ & $16.12^{\mathrm{ab}} \pm 2.407$ & $6.81^{\mathrm{a}} \pm 1.245$ \\
\hline LL & $5.45^{\mathrm{b}} \pm 0.043$ & $5.38^{c} \pm 0.035$ & $-0.07^{\mathrm{b}} \pm 0.031$ & $5.55^{\mathrm{a}} \pm 0.189$ & $0.17^{\mathrm{a}} \pm 0.208$ & $20.53^{\mathrm{ab}} \pm 4.126$ & $13.73^{\mathrm{b}} \pm 2.916$ & $6.80^{\mathrm{a}} \pm 1.578$ \\
\hline $\begin{array}{c}\text { LSD } \\
(P=0.05)\end{array}$ & 0.047 & 0.054 & 0.066 & 0.265 & 0.279 & 3.577 & 2.695 & 1.891 \\
\hline
\end{tabular}

\# s.d.: Standard deviation.

BF: biceps femoris; RF: rectus femoris; ST: semitendinosus; SS: supraspinatus; LL: longissimus lumborum.

a, b, c Column means within a treatment and between muscles with common superscripts do not differ $(P \leq 0.05)$.

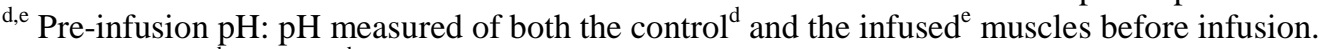

$\mathrm{pH}_{\text {difference }} \mathrm{e}^{-\mathrm{d}}$ (control $^{\mathrm{d}}$ vs. injected ${ }^{\mathrm{e}}$ ): the difference between the control and infused muscles before infusion.

${ }^{\mathrm{f}}$ Post-infusion $\mathrm{pH}: \mathrm{pH}$ measured of the infused muscles directly after infusion.

$\mathrm{pH}_{\text {difference }}^{\mathrm{f}-\mathrm{e}}$ (pre $\mathrm{e}^{\mathrm{e}}$ vs. post ${ }^{\mathrm{f}}$ infusion): the difference in $\mathrm{pH}$ between the infused muscles before and after infusion.

${ }^{\mathrm{g}}$ Pumped gain (\%) $0 \mathrm{~h}^{\mathrm{g}}$ : the amount of blend retained within the muscles directly after infusion.

${ }^{\mathrm{h}}$ Pumped gain (\%) $2 \mathrm{~h}^{\mathrm{h}}$ : the amount of blend retained within the muscles $2 \mathrm{~h}$ (resting period) after infusion.

Pumped gain difference $\mathrm{g}^{\mathrm{g}-\mathrm{h}}$ : the difference in pumped gain between the infused muscles before and after infusion.

LSD: Least significant difference $(P=0.05)$. 
The parameters used in this investigation to evaluate the colour of the raw meat, as well as the cooked samples are the $L^{*}, a^{*}, b^{*}$ and chroma values, as well as hue angle. The $L^{*}$ value gives an indication of lightness (Papadopoulos et al., 1991). Overall there was no interaction between treatment and storage time $(P>0.05)$. Although the $L^{*}$ values fluctuated during storage $(P<0.05)$, there was no noticeable pattern (Table 4). Pooled over time and processing days (Table 5), $L^{*}$ values for the raw infused muscles ranged from $38.5 \pm 2.36$ (SS) to $43.0 \pm 3.33$ (RF). These results are supported by other studies, where a similar blend was used for infusion (Papadopoulos et al., 1991). From Table 4 it is clear that the $L^{*}$ values of the infused and untreated samples differed $(P \leq 0.05)$ in only a limited number of cases. However, according to Table 5 (illustrating the overall effect), four of the infused samples had lower $(P \leq 0.05) \mathrm{L}^{*}$ values than the control samples, indicating a darker meat colour for the BF, RF, SS and LL infused samples. Lawrence et al. (2004) reported contradictory results to those of the present investigation. Lawrence et al. (2004) found that beef samples treated with either a lactate or chloride solution showed similar $\mathrm{L}^{*}$ values to those of the control. Conversely, Baublits et al. (2005) reported that the treated samples had higher L* values, thus were lighter in colour than the control samples. However, this result was obtained with the inclusion only of phosphates (Baublits et al., 2005). With the addition of sodium chloride ( $\mathrm{NaCl}$ ) to the blend, as in the present investigation, the overall colour becomes darker (Baublits et al., 2005a; b; 2006; Hoffman, 2006).

The $\mathrm{a}^{*}$ value measures the red-green range of meat with greater $\mathrm{a}^{*}$ values indicating a redder meat sample, whereas similar a* and $b^{*}$ values indicate a purple meat colour. In this investigation (Table 4) there was no interaction $(P<0.05)$ between the infusion and storage time. However, there was a tendency for both the infused and control samples to increase in redness (higher a* values) with time, indicating a deterioration as the muscles became darker. With the addition of a salt solution, the redness of muscle samples has been observed to decrease and therefore a darker sample colour is obtained (Baublits et al., 2006). And the welldocumented deterioration of fresh meat with storage, even under vacuum packaging, is the logical explanation of the decreasing meat colour. Pooled over time (Table 5), redness $\left(\mathrm{a}^{*}\right)$ was $(P \leq 0.05)$ lower in all the treated samples with means for the raw muscles ranging from $13.67 \pm 1.958$ (RF) to $15.79 \pm 1.772$ (BF). Again, the results are supported by other studies in which a similar blend was used for infusion and the effect on colour parameters determined (Papadopoulos et al., 1991). Baublits et al. (2005b) also reported control muscles to have a redder colour (higher $\mathrm{a}^{*}$ values) than the treated muscles. According to Baublits et al. (2005a), limited differences were observed between the control and muscles treated with phosphates and $\mathrm{NaCl}$. However, there was a tendency for the phosphate and salt enhanced samples to have lower a* values, suggesting the deleterious effects of salt on meat colour (Baublits et al., 2006). In the present investigation much lower $\mathrm{a}^{*}$ values were observed than reported by Baublits et al. (2005a).

The $b^{*}$ value measures the blue-yellow range of meat with a $b^{*}$ value of 0 (zero) indicating a grey appearance. With meat, greater $\mathrm{b}^{*}$ values indicate a visual description of brown (Carpenter et al., 2001). In this investigation (Table 4) there was no consistent treatment effect. However, there was a tendency for the $\mathrm{b}^{*}$ values to be lower in the treated samples. Furthermore, there was no interaction $(P<0.05)$ between the phosphate and lactate blend and storage time, and therefore the $b^{*}$ value did not change consistently during storage. The report by Papadopoulos et al. (1991), where a similar blend was used for infusion, showed comparable colour results. Pooled over time (Table 5) the means of the raw muscles treated with the blend ranged from $12.71 \pm 1.612$ (SS) to $14.90 \pm 1.995(\mathrm{ST})$. All the infused muscles had lower $\mathrm{b}^{*}$ values $(P$ $\leq 0.05$ ), indicating a lower degree of brownness. Baublits et al. (2005b) also reported control muscles to have a yellower colour (higher $b *$ values) than the treated muscles.

The saturation index is defined by higher chroma values, indicating greater saturation or vividness of colour (Baublits et al., 2005b). As illustrated in Table 4, as well as the pooled data in Table 5, the treatment had an effect $(P \leq 0.05)$ on all the muscles, with the infused muscles having lower chroma values, that is, degree of saturation. Lawrence et al. (2003) reported control muscles to have more intense red colour (higher chroma values) than the treated muscles. Baublits et al. (2005a) reported similar degrees of vividness for treated and untreated samples. This result suggests that phosphates can maintain or increase vividness. However, in combination with $\mathrm{NaCl}$ the vividness is hindered. In the present investigation, $\mathrm{NaCl}$ formed part of the blend and resulted in a poorer and less saturated raw colour. This again illustrates the negative effect of $\mathrm{NaCl}$ on beef colour (Baublits et al., 2005a). The colour change over time (Table 4) was inconsistent and no change $(P>0.05)$ in any of the muscles was observed within treatments and over time. 
Table 2 Interaction means $\left( \pm\right.$ s.d.) ${ }^{\#}$ for physical attributes of beef muscles infused with a phosphate and lactate blend and aged for 19 days

\begin{tabular}{|c|c|c|c|c|c|c|c|c|c|c|}
\hline \multirow{2}{*}{ Day } & \multicolumn{2}{|c|}{$\mathrm{pH}$} & \multicolumn{2}{|c|}{ Purge loss (\%) } & \multicolumn{2}{|c|}{ Drip loss (\%) } & \multicolumn{2}{|c|}{ Cooking loss (\%) } & \multicolumn{2}{|c|}{ WBSF (N) } \\
\hline & Control & Infused & Control & Infused & Control & Infused & Control & Infused & Control & Infused \\
\hline \multicolumn{11}{|c|}{ Biceps femoris (BF) } \\
\hline 4 & $5.45_{a b}^{b} \pm 0.039$ & $5.67_{\mathrm{bc}}{ }^{\mathrm{a}} \pm 0.046$ & $1.53_{\mathrm{b}}^{\mathrm{a}} \pm 0.487$ & $2.93_{\mathrm{b}}{ }^{\mathrm{a}} \pm 0.398$ & $0.99_{\mathrm{ab}}^{\mathrm{b}} \pm 0.314$ & $1.69_{\mathrm{a}}^{\mathrm{a}} \pm 0.334$ & $34.22_{b}{ }^{a} \pm 0.796$ & $35.42_{c}{ }^{a} \pm 1.854$ & $46.26_{c}{ }^{a} \pm 5.536$ & $33.78_{b}{ }^{b} \pm 5.110$ \\
\hline 7 & $5.45_{\mathrm{ab}}^{\mathrm{b}} \pm 0.044$ & $5.65_{b c}^{a} \pm 0.011$ & $2.80_{\mathrm{ab}}^{\mathrm{a}} \pm 0.886$ & $4.36_{\mathrm{ab}}^{\mathrm{a}} \pm 1.463$ & $1.10_{\mathrm{a}}^{\mathrm{a}} \pm 0.390$ & $1.27_{\mathrm{b}}^{\mathrm{a}} \pm 0.407$ & $41.55^{\mathrm{b}} \pm 1.017$ & $44.46_{a}^{a} \pm 1.818$ & $60.86_{a b}^{a} \pm 6.246$ & $47.19^{\mathrm{b}} \pm 7.804$ \\
\hline 10 & $5.46_{a b}^{b} \pm 0.035$ & $5.77_{a}^{a} \pm 0.100$ & $3.53_{a}^{a} \pm 1.425$ & $4.72^{a}{ }^{a} \pm 1.825$ & $0.98_{a b}{ }^{a} \pm 0.120$ & $1.12_{b c}{ }^{a} \pm 0.303$ & $41.12^{\mathrm{b}} \pm 2.263$ & $42.93_{\mathrm{ab}}{ }^{\mathrm{a}} \pm 2.013$ & $56.05_{\mathrm{abc}}{ }^{\mathrm{a}} \pm 5.497$ & $40.37_{a b}^{b} \pm 9.840$ \\
\hline 13 & $5.52_{a}^{b} \pm 0.029$ & $5.72_{a b}{ }^{a} \pm 0.064$ & $2.92_{a b}^{b} \pm 1.844$ & $4.56_{a}^{a} \pm 0.870$ & $1.26_{a}^{b} \pm 0.191$ & $1.61_{\mathrm{a}}^{\mathrm{a}} \pm 0.502$ & $41.13_{\mathrm{a}}^{\mathrm{b}} \pm 0.940$ & $44.35_{\mathrm{a}}^{\mathrm{a}} \pm 1.704$ & $61.73^{a} \pm 13.64$ & $45.95_{\mathrm{a}}^{\mathrm{b}} \pm 8.260$ \\
\hline 16 & $5.43_{b}^{b} \pm 0.046$ & $5.63_{c}{ }^{a} \pm 0.095$ & $4.25_{a}^{a} \pm 1.591$ & $5.37_{\mathrm{a}}^{\mathrm{a}} \pm 1.097$ & $0.70_{b}^{a} \pm 0.121$ & $0.81_{c}^{a} \pm 0.122$ & $40.65^{a} \pm 0.796$ & $42.00_{\mathrm{b}}^{\mathrm{a}} \pm 2.706$ & $51.44_{b c}{ }^{a} \pm 11.23$ & $33.69^{b}{ }^{b} \pm 7.859$ \\
\hline 19 & $5.34_{c}^{b} \pm 0.038$ & $5.66_{b c}^{a} \pm 0.079$ & $3.99_{a}^{a} \pm 1.184$ & $4.03_{a b}{ }^{a} \pm 1.054$ & $1.13_{\mathrm{a}}^{\mathrm{a}} \pm 0.160$ & $1.10_{\mathrm{bc}}{ }^{\mathrm{a}} \pm 0.213$ & $40.86_{a}^{a} \pm 1.483$ & $41.45_{\mathrm{b}}^{\mathrm{a}} \pm 2.142$ & $47.04_{c}{ }^{a} \pm 12.05$ & $32.42_{\mathrm{b}}^{\mathrm{b}} \pm 4.560$ \\
\hline \multicolumn{11}{|c|}{ Rectus femoris (RF) } \\
\hline 4 & $5.42_{b c}^{b} \pm 0.034$ & $5.65_{b}{ }^{a} \pm 0.081$ & $4.54_{a}^{a} \pm 2.089$ & $5.87_{\mathrm{a}}^{\mathrm{a}} \pm 1.496$ & $1.39_{\mathrm{ab}}^{\mathrm{b}} \pm 0.290$ & $2.06_{a}^{a} \pm 0.339$ & $37.32_{c}^{b} \pm 2.275$ & $40.13_{b}{ }^{a} \pm 2.162$ & $58.68_{\mathrm{ab}}{ }^{\mathrm{a}} \pm 11.82$ & $41.53_{a b}^{b} \pm 5.809$ \\
\hline 7 & $5.46_{b}^{b} \pm 0.063$ & $5.79_{a}^{a} \pm 0.116$ & $3.92_{a}^{a} \pm 2.007$ & $5.47_{a}^{a} \pm 1.650$ & $1.24_{b}^{b} \pm 0.216$ & $1.57_{\mathrm{b}}^{\mathrm{a}} \pm 0.275$ & $40.36_{b}^{b} \pm 1.739$ & $42.75_{\mathrm{a}}^{\mathrm{a}} \pm 2.617$ & $61.00^{a} \pm 13.36$ & $42.61_{\mathrm{a}}^{\mathrm{b}} \pm 8.977$ \\
\hline 10 & $5.50_{\mathrm{ab}}^{\mathrm{b}} \pm 0.058$ & $5.78_{a}^{a} \pm 0.087$ & $4.44_{a}^{a} \pm 1.195$ & $5.43_{a}^{a} \pm 1.453$ & $1.23_{\mathrm{b}}{ }^{\mathrm{a}} \pm 0.117$ & $1.21_{c}^{\mathrm{a}} \pm 0.152$ & $40.33_{\mathrm{b}}^{\mathrm{b}} \pm 1.003$ & $42.06_{\mathrm{a}}^{\mathrm{a}} \pm 1.502$ & $52.96_{\mathrm{abc}}{ }^{\mathrm{a}} \pm 4.048$ & $36.72_{a b c}{ }^{b} \pm 7.977$ \\
\hline 13 & $5.58_{a}^{b} \pm 0.082$ & $5.79_{a}^{a} \pm 0.116$ & $3.72_{a}^{b} \pm 1.308$ & $6.64_{a}^{a} \pm 1.705$ & $1.60_{\mathrm{a}}^{\mathrm{a}} \pm 0.190$ & $1.41_{b c}{ }^{a} \pm 0.220$ & $40.03_{b}^{b} \pm 1.557$ & $41.79_{a}^{a} \pm 1.697$ & $50.08_{\mathrm{bc}}{ }^{\mathrm{a}} \pm 8.457$ & $32.72_{a b c}{ }^{b} \pm 2.489$ \\
\hline 16 & $5.47_{b}^{b} \pm 0.045$ & $5.77_{\mathrm{a}}^{\mathrm{a}} \pm 0.099$ & $4.55_{a}^{a} \pm 1.126$ & $5.80_{\mathrm{a}}{ }^{\mathrm{a}} \pm 1.466$ & $1.16_{\mathrm{b}}^{\mathrm{a}} \pm 0.137$ & $1.11_{c}^{\mathrm{a}} \pm 0.315$ & $42.44_{a}^{a} \pm 1.658$ & $43.399^{a} \pm 2.615$ & $51.52_{\mathrm{abc}}{ }^{\mathrm{a}} \pm 8.748$ & $27.44_{C}{ }^{b} \pm 7.322$ \\
\hline 19 & $5.36_{c}^{b} \pm 0.058$ & $5.63_{b}{ }^{a} \pm 0.090$ & $4.26_{a}^{b} \pm 0.871$ & $6.50_{a}^{a} \pm 1.156$ & $1.18_{b}{ }^{a} \pm 0.290$ & $1.27_{\mathrm{bc}}{ }^{\mathrm{a}} \pm 0.187$ & $40.84_{a b}{ }^{a} \pm 2.471$ & $42.05_{a}^{a} \pm 2.261$ & $48.44_{c}{ }^{a} \pm 9.766$ & $35.32_{\mathrm{abc}}{ }^{\mathrm{b}} \pm 8.998$ \\
\hline \multicolumn{11}{|c|}{ Semitendinosus (ST) } \\
\hline 4 & $5.45_{b}^{b} \pm 0.030$ & $5.73_{\mathrm{a}}{ }^{\mathrm{a}} \pm 0.112$ & $2.67_{b}^{b} \pm 1.100$ & $6.61_{\mathrm{a}}^{\mathrm{a}} \pm 1.040$ & $0.98_{\mathrm{ab}}^{\mathrm{b}} \pm 0.507$ & $2.75_{a}^{a} \pm 0.392$ & $39.02_{c}^{b} \pm 1.330$ & $41.74_{b c}{ }^{a} \pm 2.277$ & $86.23_{\mathrm{ab}}{ }^{\mathrm{a}} \pm 11.88$ & $51.48_{a}^{b} \pm 9.642$ \\
\hline 7 & $5.43_{b}^{b} \pm 0.028$ & $5.65_{a b}{ }^{a} \pm 0.135$ & $4.47_{\mathrm{a}}^{\mathrm{b}} \pm 1.27$ & $7.77_{\mathrm{a}}^{\mathrm{a}} \pm 1.224$ & $0.85_{b}^{b} \pm 0.327$ & $1.42_{b}^{a} \pm 0.358$ & $42.11_{\mathrm{a}}^{\mathrm{a}} \pm 0.914$ & $43.64^{a} \pm 1.845$ & $92.64^{a} \pm 14.43$ & $48.56_{a b}^{b} \pm 8.630$ \\
\hline 10 & $5.48_{\mathrm{ab}}^{\mathrm{b}} \pm 0.053$ & $5.65_{a b}^{a} \pm 0.084$ & $3.72_{a b}{ }^{b} \pm 1.486$ & $8.08_{a}{ }^{a} \pm 1.633$ & $0.79_{\mathrm{b}}^{\mathrm{a}} \pm 0.297$ & $0.84_{c}^{\mathrm{a}} \pm 0.168$ & $40.17_{\mathrm{bc}}{ }^{\mathrm{a}} \pm 1.384$ & $40.42_{c}{ }^{a} \pm 2.894$ & $79.10_{\mathrm{bc}}{ }^{\mathrm{a}} \pm 19.42$ & $48.99_{a}^{b} \pm 15.88$ \\
\hline 13 & $5.55_{a}^{b} \pm 0.041$ & $5.72_{a}^{a} \pm 0.102$ & $3.65_{\mathrm{ab}}^{\mathrm{b}} \pm 1.805$ & $6.88_{a}{ }^{a} \pm 1.312$ & $1.27_{\mathrm{a}}^{\mathrm{a}} \pm 0.233$ & $1.58_{b}{ }^{a} \pm 0.481$ & $41.70_{a b}{ }^{a} \pm 0.845$ & $42.66_{\mathrm{ab}}{ }^{\mathrm{a}} \pm 1.542$ & $82.35_{\mathrm{b}}^{\mathrm{a}} \pm 11.85$ & $47.68_{a b}^{b} \pm 13.18$ \\
\hline 16 & $5.41_{b c}^{\mathrm{b}} \pm 0.050$ & $5.63_{b}{ }^{a} \pm 0.126$ & $5.16^{b}{ }^{b} \pm 1.866$ & $6.93_{\mathrm{a}}^{\mathrm{a}} \pm 1.648$ & $0.73_{b}^{a} \pm 0.116$ & $0.96_{c}^{a} \pm 0.298$ & $40.69_{a b}{ }^{a} \pm 1.100$ & $41.45_{b c}{ }^{a} \pm 2.340$ & $80.09_{b c}{ }^{a} \pm 19.82$ & $50.46_{a}^{b} \pm 14.99$ \\
\hline 19 & $5.33_{c}^{b} \pm 0.053$ & $5.58_{b}{ }^{a} \pm 0.092$ & $5.21_{a}^{\mathrm{b}} \pm 2.634$ & $7.97_{a}^{a} \pm 2.118$ & $0.90_{b}{ }^{a} \pm 0.076$ & $1.04_{c}^{a} \pm 0.118$ & $41.11_{\mathrm{ab}}{ }^{\mathrm{a}} \pm 0.966$ & $42.60_{\mathrm{ab}}^{\mathrm{a}}{ }^{\mathrm{a}} \pm 1.633$ & $70.93_{c}{ }^{a} \pm 7.007$ & $38.77_{b}^{b} \pm 4.275$ \\
\hline
\end{tabular}


Table 2 (continued) Interaction means ( \pm s.d.) $)^{\#}$ for physical attributes of beef muscles infused with a phosphate and lactate blend and aged for 19 days

\begin{tabular}{|c|c|c|c|c|c|c|c|c|c|c|}
\hline \multirow{2}{*}{ Day } & \multicolumn{2}{|c|}{$\mathrm{pH}$} & \multicolumn{2}{|c|}{ Purge loss (\%) } & \multicolumn{2}{|c|}{ Drip loss (\%) } & \multicolumn{2}{|c|}{ Cooking loss (\%) } & \multicolumn{2}{|c|}{ WBSF (N) } \\
\hline & Control & Infused & Control & Infused & Control & Infused & Control & Infused & Control & Infused \\
\hline \multicolumn{11}{|c|}{ Supraspinatus (SS) } \\
\hline 4 & $5.51_{b c}^{b} \pm 0.026$ & $5.81_{b c}{ }^{a} \pm 0.097$ & $2.16_{b}^{b} \pm 0.572$ & $5.09_{\mathrm{b}}^{\mathrm{a}} \pm 1.282$ & $0.72_{b}^{b} \pm 0.116$ & $1.26_{\mathrm{ab}}{ }^{\mathrm{a}} \pm 0.402$ & $40.45^{b} \pm 1.087$ & $42.11_{c}{ }^{a} \pm 1.785$ & $69.71_{\mathrm{ab}}^{\mathrm{a}} \pm 8.950$ & $54.21_{\mathrm{a}}^{\mathrm{b}} \pm 7.283$ \\
\hline 7 & $5.60_{\mathrm{a}}^{\mathrm{b}} \pm 0.051$ & $5.88_{a b}{ }^{a} \pm 0.065$ & $3.17_{a b}^{b} \pm 0.663$ & $5.28_{\mathrm{ab}}^{\mathrm{a}} \pm 1.134$ & $0.79_{b}^{b} \pm 0.172$ & $1.15_{\mathrm{ab}}^{\mathrm{a}} \pm 0.393$ & $45.64_{a}{ }^{a} \pm 1.739$ & $46.39^{a}{ }^{a} \pm 2.201$ & $77.37_{\mathrm{a}}^{\mathrm{a}} \pm 10.87$ & $47.23_{\mathrm{ab}}^{\mathrm{b}} \pm 3.842$ \\
\hline 10 & $5.64_{a}^{b} \pm 0.033$ & $5.95^{a}{ }^{a} \pm 0.119$ & $3.45_{a b}^{b} \pm 0.800$ & $6.11_{\mathrm{ab}}^{\mathrm{a}} \pm 1.572$ & $0.85_{b}{ }^{a} \pm 0.100$ & $0.95_{\mathrm{b}}^{\mathrm{a}} \pm 0.292$ & $45.04_{a b}^{a} \pm 0.559$ & $45.11_{\mathrm{ab}}^{\mathrm{a}} \pm 1.423$ & $70.80_{\mathrm{ab}}{ }^{\mathrm{a}} \pm 9.375$ & $48.11_{\mathrm{ab}}^{\mathrm{b}} \pm 3.219$ \\
\hline 13 & $5.59_{a b}^{b} \pm 0.079$ & $5.84_{\mathrm{bc}}{ }^{\mathrm{a}} \pm 0.118$ & $3.15_{a b}^{b} \pm 1.151$ & $6.20_{\mathrm{ab}}^{\mathrm{a}} \pm 1.500$ & $1.52_{a}{ }^{a} \pm 0.222$ & $1.47_{\mathrm{a}}^{\mathrm{a}} \pm 0.534$ & $43.72_{\mathrm{b}}{ }^{\mathrm{a}} \pm 1.182$ & $44.46_{\mathrm{b}}^{\mathrm{a}} \pm 1.194$ & $63.57_{\mathrm{b}}^{\mathrm{a}} \pm 8.859$ & $47.03_{\mathrm{ab}}^{\mathrm{b}} \pm 5.649$ \\
\hline 16 & $5.60_{\mathrm{a}}^{\mathrm{b}} \pm 0.047$ & $5.86_{\mathrm{bc}}{ }^{\mathrm{a}} \pm 0.080$ & $3.64_{a b}{ }^{b} \pm 1.660$ & $6.25_{\mathrm{ab}}^{\mathrm{a}} \pm 1.395$ & $0.86_{b}{ }^{a} \pm 0.195$ & $1.03_{\mathrm{b}}{ }^{\mathrm{a}} \pm 0.214$ & $44.55_{\mathrm{ab}}^{\mathrm{a}} \pm 1.459$ & $43.83^{a}{ }^{a} \pm 1.633$ & $65.22_{\mathrm{b}}^{\mathrm{a}} \pm 3.509$ & $45.95_{a b}^{b} \pm 8.342$ \\
\hline 19 & $5.48_{c}{ }^{b} \pm 0.067$ & $5.78_{c}{ }^{a} \pm 0.076$ & $4.15_{a}^{b} \pm 1.753$ & $6.83_{a}^{a} \pm 1.955$ & $0.96_{b}{ }^{a} \pm 0.137$ & $1.15_{a b}{ }^{a} \pm 0.143$ & $43.83_{b}{ }^{a} \pm 2.138$ & $44.14_{b}{ }^{a} \pm 1.585$ & $66.11_{\mathrm{b}}{ }^{\mathrm{a}} \pm 6.237$ & $41.04_{b}{ }^{b} \pm 4.975$ \\
\hline \multicolumn{11}{|c|}{ Longissimus lumborum (LL) } \\
\hline 4 & $5.40_{\mathrm{bc}}^{\mathrm{b}} \pm 0.026$ & $5.55_{c}^{a} \pm 0.077$ & $2.90_{b}^{b} \pm 0.781$ & $4.966_{b}{ }^{a} \pm 1.013$ & $1.19_{b}{ }^{\mathrm{a}} \pm 0.171$ & $1.22_{\mathrm{b}}^{\mathrm{a}} \pm 0.385$ & $40.11_{\mathrm{abc}}{ }^{\mathrm{a}} \pm 1.138$ & $40.022_{b c}{ }^{a} \pm 2.043$ & $79.04_{\mathrm{a}}^{\mathrm{a}} \pm 16.67$ & $54.73_{\mathrm{a}}^{\mathrm{b}} \pm 9.705$ \\
\hline 7 & $5.47_{\mathrm{ab}}^{\mathrm{b}} \pm 0.018$ & $5.63_{\mathrm{bc}}{ }^{\mathrm{a}} \pm 0.077$ & $6.04_{a}^{a} \pm 1.128$ & $6.39_{\mathrm{ab}}^{\mathrm{a}} \pm 1.434$ & $1.91_{\mathrm{a}}^{\mathrm{a}} \pm 0.450$ & $1.94_{a}^{a} \pm 0.349$ & $40.90_{\mathrm{ab}}^{\mathrm{a}} \pm 1.436$ & $41.71_{a}^{a} \pm 2.567$ & $75.78_{a}^{a} \pm 20.17$ & $49.21_{\mathrm{ab}}^{\mathrm{b}} \pm 19.90$ \\
\hline 10 & $5.45_{\mathrm{ab}}^{\mathrm{b}} \pm 0.057$ & $5.70_{\mathrm{ab}}^{\mathrm{a}} \pm 0.071$ & $5.76_{a}^{a} \pm 1.296$ & $7.01_{\mathrm{a}}^{\mathrm{a}} \pm 0.893$ & $1.01_{b}{ }^{a} \pm 0.438$ & $1.14_{\mathrm{b}}^{\mathrm{a}} \pm 0.454$ & $39.83_{\mathrm{bc}}{ }^{\mathrm{a}} \pm 1.249$ & $39.74_{b c}{ }^{a} \pm 1.769$ & $63.96_{\mathrm{b}}^{\mathrm{a}} \pm 10.56$ & $39.29_{\mathrm{bc}}^{\mathrm{b}} \pm 17.44$ \\
\hline 13 & $5.52_{a}^{b} \pm 0.046$ & $5.73_{a}{ }^{a} \pm 0.091$ & $5.28_{\mathrm{a}}^{\mathrm{a}} \pm 1.256$ & $6.15_{\mathrm{ab}}^{\mathrm{a}} \pm 0.819$ & $1.28_{\mathrm{b}}{ }^{\mathrm{a}} \pm 0.224$ & $1.40_{\mathrm{b}}^{\mathrm{a}} \pm 0.249$ & $38.54_{c}{ }^{a} \pm 1.528$ & $39.38_{\mathrm{c}}{ }^{\mathrm{a}} \pm 2.604$ & $54.93_{\mathrm{b}}{ }^{\mathrm{a}} \pm 11.22$ & $39.12_{c}^{b} \pm 17.98$ \\
\hline 16 & $5.42_{b}^{b} \pm 0.018$ & $5.57_{c}^{a} \pm 0.088$ & $5.62_{a}^{a} \pm 1.748$ & $7.12^{a}{ }^{a} \pm 1.845$ & $1.06_{b}{ }^{a} \pm 0.218$ & $1.34_{b}{ }^{a} \pm 0.373$ & $41.14_{\mathrm{ab}}^{\mathrm{a}} \pm 1.261$ & $41.14_{\mathrm{ab}}^{\mathrm{a}} \pm 1.862$ & $58.69_{\mathrm{b}}^{\mathrm{a}} \pm 11.44$ & $33.65^{b} \pm 12.44$ \\
\hline 19 & $5.33_{c}^{b} \pm 0.058$ & $5.57_{c}^{\mathrm{a}} \pm 0.073$ & $5.37_{\mathrm{a}}^{\mathrm{a}} \pm 1.097$ & $6.20_{\mathrm{ab}}^{\mathrm{a}} \pm 1.726$ & $0.97_{b}{ }^{a} \pm 0.164$ & $1.16_{\mathrm{b}}{ }^{\mathrm{a}} \pm 0.242$ & $41.68^{a}{ }^{a} \pm 1.155$ & $41.36_{a b}{ }^{a} \pm 0.958$ & $58.56_{\mathrm{b}}{ }^{\mathrm{a}} \pm 15.25$ & $30.47_{c}^{b} \pm 8.662$ \\
\hline $\begin{array}{c}\text { LSD } \\
P=0.05\end{array}$ & \multicolumn{2}{|c|}{1.968} & \multicolumn{2}{|c|}{1.568} & \multicolumn{2}{|c|}{0.324} & \multicolumn{2}{|c|}{1.654} & \multicolumn{2}{|c|}{10.01} \\
\hline
\end{tabular}

a, b, c Column means between days within a treatment and within a muscle with common subscripts do not differ $(P \leq 0.05)$

${ }^{a}$ Row means between treatments within an attribute with common superscripts do not differ $(P \leq 0.05)$.

LSD: least significant difference $(P=0.05)$. 
Table 3 Summary of means ( \pm s.d.) $)^{\#}$ for physical attributes of different beef muscles (pooled) infused with a phosphate and lactate blend and aged for 19 days

\begin{tabular}{|c|c|c|c|c|c|c|c|c|c|c|}
\hline \multirow{2}{*}{ Muscle } & \multicolumn{2}{|c|}{$\mathrm{pH}$} & \multicolumn{2}{|c|}{ Purge loss (\%) } & \multicolumn{2}{|c|}{ Drip loss (\%) } & \multicolumn{2}{|c|}{ Cooking loss (\%) } & \multicolumn{2}{|c|}{ WBSF (N) } \\
\hline & Control & Infused & Control & Infused & Control & Infused & Control & Infused & Control & Infused \\
\hline $\mathrm{BF}$ & $5.44_{b}^{b} \pm 0.065$ & $5.68_{c}{ }^{a} \pm 0.083$ & $3.17_{c}^{b} \pm 1.523$ & $4.33_{c}^{a} \pm 1.347$ & $1.03_{\mathrm{b}}^{\mathrm{b}} \pm 0.288$ & $1.24_{b c}{ }^{a} \pm 0.428$ & $39.92_{c}^{b} \pm 2.876$ & $41.77_{\mathrm{b}}^{\mathrm{a}} \pm 3.634$ & $53.90_{c}^{a} \pm 10.88$ & $38.90_{b c}^{b} \pm 9.21$ \\
\hline $\mathrm{RF}$ & $5.46_{b}^{b} \pm 0.088$ & $5.73_{\mathrm{b}}{ }^{\mathrm{a}} \pm 0.113$ & $4.24_{b}^{b} \pm 1.428$ & $5.95_{b}{ }^{a} \pm 1.465$ & $1.30_{\mathrm{a}}^{\mathrm{a}} \pm 0.254$ & $1.42^{a}{ }^{a} \pm 0.390$ & $40.22_{b c}^{b} \pm 2.302$ & $42.03_{b}{ }^{a} \pm 2.263$ & $53.78_{c}{ }^{a} \pm 10.19$ & $36.06^{b} \pm 8.53$ \\
\hline ST & $5.44_{b}^{b} \pm 0.079$ & $5.66_{c}^{a} \pm 0.115$ & $4.16_{b}^{b} \pm 1.882$ & $7.37_{\mathrm{a}}^{\mathrm{a}} \pm 1.541$ & $0.92_{b}^{b} \pm 0.321$ & $1.43_{a}^{a} \pm 0.728$ & $40.80^{b}{ }^{b} \pm 1.454$ & $42.09_{b}{ }^{a} \pm 2.238$ & $82.20_{a}^{a} \pm 15.31$ & $47.63^{\mathrm{b}} \pm 11.79$ \\
\hline SS & $5.57_{\mathrm{a}}^{\mathrm{b}} \pm 0.075$ & $5.86_{a}^{a} \pm 0.103$ & $3.29_{c}^{b} \pm 1.279$ & $5.96_{b}{ }^{a} \pm 1.511$ & $0.95_{b}^{b} \pm 0.307$ & $1.17_{c}^{a} \pm 0.376$ & $43.72^{a}{ }^{a} \pm 2.241$ & $44.34_{a}^{a} \pm 2.028$ & $68.79_{\mathrm{b}}^{\mathrm{a}} \pm 9.00$ & $47.26_{a}^{b} \pm 6.67$ \\
\hline LL & $5.43_{b}^{b} \pm 0.073$ & $5.62_{d}^{a} \pm 0.102$ & $5.16_{a}^{b} \pm 1.569$ & $6.31_{\mathrm{b}}{ }^{\mathrm{a}} \pm 1.446$ & $1.22_{\mathrm{a}}^{\mathrm{a}} \pm 0.412$ & $1.35_{\mathrm{ab}}{ }^{\mathrm{a}} \pm 0.416$ & $40.35_{b c}{ }^{a} \pm 1.593$ & $40.56_{c}^{a} \pm 2.093$ & $65.70_{\mathrm{b}}^{\mathrm{a}} \pm 16.66$ & $41.08_{b}^{b} \pm 16.30$ \\
\hline $\begin{array}{c}\text { LSD } \\
P=0.05\end{array}$ & \multicolumn{2}{|c|}{0.033} & \multicolumn{2}{|c|}{0.640} & \multicolumn{2}{|c|}{0.132} & \multicolumn{2}{|c|}{0.668} & \multicolumn{2}{|c|}{4.083} \\
\hline
\end{tabular}

BF: biceps femoris; RF: rectus femoris; ST: semitendinosus; SS: supraspinatus; LL: longissimus lumborum. a, b, c Column means within a treatment with common subscripts do not differ $(P \leq 0.05)$.

a, b Row means within an attribute and between treatments with common superscripts do not differ $(P \leq 0.05)$.

LSD: least significant difference $(P=0.05)$. 
According to Baublits et al. (2005a), the inclusion of phosphate-based solutions increases or results in similar hue angles to those of the control samples. However, with the addition of $\mathrm{NaCl}$ the hue angle decreased, indicating a deterioration of redness when $\mathrm{NaCl}$ is included. In the present investigation the infusion had no $(P>0.05)$ effect on the hue angle (Tables 4 and 5$)$ and with time the pattern was inconsistent in both the control and infused samples. According to the pooled data (Table 5), four of the five muscles had similar hue angles $(P>0.05)$. Only the infused ST had a higher hue angle. This is supported by other research studies, which reported higher hue angles for infused muscles (Baublits et al., 2005b; Lawrence et al., 2003).

The results on the instrumental colour of the cooked samples are illustrated in Table 6. In general, the blend did not affect the muscle lightness $\left(\mathrm{L}^{*}\right)$ of the cooked muscles significantly (Table 6). Overall, however, the $\mathrm{L}^{*}$ values of the infused samples were higher $(P \leq 0.05)$ and the infused samples were therefore slightly lighter in appearance (Table 7). No pattern $(P>0.05)$ over time with regard to lightness was visible within treatments (Table 6).

The $\mathrm{a}^{*}$ value showed no $(P>0.05)$ effect with regard to the treatment (Table 6). The change within treatment over time indicated no pattern and suggests no $(P>0.05)$ change over time (Table 6$)$. Overall the infused samples were generally $(P \leq 0.05)$ lower in cooked a* colour (Table 7). The $\mathrm{b}^{*}$ and chroma values followed similar patterns, that is, lower $(P \leq 0.05)$ values in the infused muscles.

With the hue angle calculations (Table 7), the infused muscles had slightly higher values than the control samples. However, only the infused ST and LL samples were higher $(P \leq 0.05)$. Thus, overall the infused cooked samples appeared redder. Other research reported higher hue angles for infused muscles (Lawrence et al., 2003; Baublits et al., 2005a; b; 2006).

Lactate has been described as a 'colour-stabilizer' in fresh beef, minimizing surface colour change by producing a dark-coloured pigment that is stable during retailing (Lawrence et al., 2004). Maca et al. (1999) concluded that $\mathrm{NaLac}$ had a protective effect on the meat colour and acted as a stabiliser. This was observed in the treated muscles of this investigation, that is, they had a slightly redder colour than the control sample. Research into the mechanism of lactate-induced beef colour stability indicates that added lactate promotes maintenance of ferrous Mb redox forms (Kim et al., 2006; Mancini \& Ramanathan, 2007; Suman et al., 2009). In conclusion, colour values fluctuated during the storage of raw and cooked beef over the 19 days and no clear pattern could be found.

The proximate chemical composition values were determined using the muscles samples taken from Day 4 and the results are presented in Table 8. The mineral content of the muscles is shown in Table 9.

The selected beef muscles were compared for percentage moisture, protein, lipid and ash content (Table 8). The proximate chemical composition of the control sample of this investigation is similar to that reported for beef (Sayed et al., 1999; Hoffman, 2006). The results of the infused muscles presented in Table 8 are in agreement with what is expected when a solution of water and several minerals, such as phosphates, potassium and sodium is infused, into beef muscle, that is, an increase in moisture and ash content and a decrease in protein and lipid content (Hoffman, 2006).

The percentage moisture was influenced $(P \leq 0.05)$ by the infusion of the phosphate and lactate blend three of the five muscles had increased moisture content. The protein content of the infused BF and RF muscles was lower $(P \leq 0.05)$ than that of the control samples. The control and infused muscles were very similar in fat content, except for the BF muscles, where the expected lower fat content of enhanced meat was obtained (Hoffman, 2006) with the addition of a water-based solution. Because the infusion blend contained several minerals such as potassium and sodium, differences $(P \leq 0.05)$ in the ash content between the treated and control muscles were expected, as shown in Table 8.

The muscles differed in proximate composition in this investigation (Table 8). However, the differences between muscles within treatments showed no definite pattern. It was observed that the BF muscle had the lowest moisture content and highest fat content compared with the other beef muscles. Other studies have reported this inverse relationship (Delgado et al., 2005).

The results of this investigation indicated differences $(P \leq 0.05)$ in the mineral composition (Table 9) between muscles. Several other studies indicated differences in mineral content among various muscles (Schönfeldt \& Welgemoed, 1996; Hoffman, 2006). 
Table 4 Means $( \pm \text { s.d.) })^{\#}$ for colour attributes of the raw beef muscles infused with a phosphate and lactate blend and aged for 19 days

\begin{tabular}{|c|c|c|c|c|c|c|c|c|c|c|}
\hline \multirow{2}{*}{ Day } & \multicolumn{2}{|c|}{ Raw L* } & \multicolumn{2}{|c|}{ Raw a* } & \multicolumn{2}{|c|}{ Raw b* } & \multicolumn{2}{|c|}{ Raw chroma } & \multicolumn{2}{|c|}{ Raw hue angle } \\
\hline & Control & Infused & ontrol & Infused & Control & Infused & Control & Infused & Control & Infused \\
\hline \multicolumn{11}{|c|}{ Biceps femoris (BF) } \\
\hline 4 & $39.29_{a}^{a} \pm 1.758$ & $39.90_{\mathrm{a}}{ }^{\mathrm{a}} \pm 1.741$ & $15.33_{\mathrm{e}}^{\mathrm{a}} \pm 1.470$ & $14.19_{\mathrm{d}}^{\mathrm{a}} \pm 1.297$ & $13.57_{b c}{ }^{a} \pm 1.585$ & $12.70_{\mathrm{c}}^{\mathrm{a}} \pm 1.535$ & $20.50_{c}{ }^{a} \pm 2.053$ & $19.08_{\mathrm{c}}{ }^{\mathrm{a}} \pm 1.823$ & $41.46_{\mathrm{a}}^{\mathrm{a}} \pm 1.940$ & $41.46_{a}^{a} \pm 2.577$ \\
\hline 7 & $39.78_{a}^{a} \pm 1.903$ & $39.35^{a}{ }^{a} \pm 1.387$ & $16.06_{\mathrm{de}}^{\mathrm{a}} \pm 1.814$ & $15.65_{\mathrm{bc}}^{\mathrm{a}} \pm 1.742$ & $13.17_{c}^{a} \pm 2.674$ & $14.46_{\mathrm{ab}}^{\mathrm{a}} \pm 1.643$ & $20.87_{c}^{a} \pm 2.834$ & $21.36_{\mathrm{ab}}^{\mathrm{a}} \pm 2.260$ & $8.88^{{ }^{b}} \pm 4.039$ & $42.61_{a}^{a} \pm 2.193$ \\
\hline 10 & $39.94_{a}{ }^{a} \pm 2.637$ & $38.51_{a b}{ }^{a} \pm 2.285$ & $16.95_{\mathrm{cd}}^{\mathrm{a}} \pm 2.006$ & $14.97 \mathrm{~cd} \pm 0.778$ & $15.05_{\mathrm{ab}}^{\mathrm{a}} \pm 2.173$ & $13.03_{b c}{ }^{b} \pm 1.817$ & $22.69_{\mathrm{b}}{ }^{\mathrm{a}} \pm 2.874$ & $19.91_{b c}{ }^{b} \pm 1.440$ & $1.53_{a}^{a} \pm 1.605$ & $40.88^{a}{ }^{a} \pm 3.796$ \\
\hline 13 & $40.93_{a}^{a} \pm 2.601$ & $39.02_{a \mathrm{a}}^{\mathrm{a}} \pm 1.950$ & $18.34_{a^{a}}{ }^{a} \pm 1.377$ & $16.26_{a b}^{b} \pm 1.432$ & $16.35^{a}{ }^{a} \pm 1.080$ & $14.39_{a b}^{b} \pm 1.575$ & $24.58^{a}{ }^{a} \pm 1.637$ & $21.75^{\mathrm{b}} \pm 1.899$ & $1.71_{a}{ }^{a} \pm 1.445$ & $41.61_{\mathrm{a}}^{\mathrm{a}} \pm 2.781$ \\
\hline 16 & $40.60_{a}^{a} \pm 1.796$ & $40.52^{a}{ }^{a} \pm 2.036$ & $19.48_{a}^{a} \pm 1.206$ & $17.44_{a}^{b} \pm 2.325$ & $15.95_{a}{ }^{a} \pm 1.047$ & $14.66_{a}{ }^{a} \pm 1.439$ & $25.20_{\mathrm{a}}{ }^{\mathrm{a}} \pm 1.356$ & $22.80^{\mathrm{b}} \pm 2.610$ & $39.29^{a} \pm 1.939$ & $40.11_{\mathrm{a}}^{\mathrm{a}} \pm 2.022$ \\
\hline 19 & $39.43_{a}^{a} \pm 1.157$ & $36.94_{b}{ }^{b} \pm 2.049$ & $18.10_{\mathrm{bc}}{ }^{\mathrm{a}} \pm 0.987$ & $16.26_{a b}{ }^{b} \pm 1.223$ & $16.16_{a}^{a} \pm 1.236$ & $14.35_{a b}{ }^{b} \pm 1.777$ & $24.30_{\mathrm{ab}}{ }^{\mathrm{a}} \pm 1.444$ & $21.76_{a}^{\mathrm{b}} \pm$ & $41.66_{a}^{a} \pm 1.565$ & $41.34_{a}{ }^{a} \pm$ \\
\hline \multicolumn{11}{|c|}{ Rectus femoris (RF) } \\
\hline 4 & $49.74^{a}=$ & $48.01_{a}{ }^{a}=$ & 1 & {$[1$ a } & & $=\sigma^{\mathrm{a}}$ & 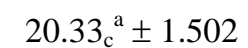 & $a_{0}{ }^{2}$ & $44^{a}$ & $9.65_{a}^{a} \pm 1.518$ \\
\hline 7 & $43.48_{b}{ }^{a} \pm 2.746$ & $41.07_{\mathrm{b}}^{\mathrm{b}} \pm 2.747$ & $14.41_{c_{d}}{ }^{a} \pm 1.587$ & $12.72_{\mathrm{cd}}^{\mathrm{b}} \pm 2.129$ & $14.71_{\mathrm{b}}{ }^{\mathrm{a}} \pm 1.538$ & $12.06_{b}^{b} \pm 2.102$ & $0.68_{c}{ }^{a} \pm 1.800$ & $17.65^{b} \pm 2.602$ & $45.56_{\mathrm{b}}^{\mathrm{a}} \pm 3.479$ & $43.32^{a}{ }^{a} \pm 4.634$ \\
\hline 10 & $42.95_{b}{ }^{a} \pm 1.749$ & $42.04_{b}{ }^{a} \pm 1.487$ & $15.06_{b c}{ }^{a} \pm 1.666$ & $13.84_{a b c}{ }^{a} \pm 2.803$ & $15.54_{a b}{ }^{a} \pm 1.164$ & $13.20^{b}{ }^{b} \pm 1.594$ & $21.67_{b c}{ }^{a} \pm 1.797$ & $19.19_{\mathrm{bc}}^{\mathrm{b}} \pm 2.958$ & $45.99_{\mathrm{b}}^{\mathrm{a}} \pm 2.560$ & $44.40_{\mathrm{b}}^{\mathrm{a}} \pm 4.230$ \\
\hline 13 & $44.66_{b}{ }^{a} \pm 3.087$ & $42.75_{b}^{a} \pm 3.189$ & $16.32_{a b}{ }^{a} \pm 1.371$ & $14.25_{a b}^{b} \pm 1.916$ & $15.59_{\mathrm{ab}}^{\mathrm{a}} \pm 1.137$ & $13.43_{\mathrm{ab}}^{\mathrm{b}} \pm 1.896$ & $22.61_{a b}{ }^{a} \pm 1.187$ & $19.64_{\mathrm{ab}}^{\mathrm{b}} \pm 2.513$ & $43.73_{b}{ }^{\mathrm{a}} \pm 3.291$ & $43.21_{b}{ }^{a} \pm 2.737$ \\
\hline 16 & $45.21_{\mathrm{b}}^{\mathrm{a}} \pm 3.239$ & $42.19^{b} \pm 2.175$ & $16.01_{\mathrm{ab}}^{\mathrm{a}} \pm 1.229$ & $13.64_{\mathrm{bcd}} \mathrm{b} \pm 1.280$ & $15.87_{a b}^{a} \pm 1.190$ & $13.34_{\mathrm{ab}}^{\mathrm{b}} \pm 1.705$ & $22.61_{a b}{ }^{a} \pm 0.805$ & $19.14_{b c}{ }^{b} \pm 1.886$ & $44.79_{b}{ }^{a} \pm 3.822$ & $44.33_{\mathrm{b}}{ }^{\mathrm{a}} \pm 2.869$ \\
\hline 19 & $43.73_{\mathrm{b}}{ }^{\mathrm{a}} \pm 2.959$ & $41.52^{a}{ }^{a} \pm 3.235$ & $16.89_{\mathrm{a}}^{\mathrm{a}} \pm 1.296$ & $15.05^{\mathrm{b}} \pm 1.772$ & $16.51_{a}{ }^{a} \pm 1.790$ & $14.78_{\mathrm{a}}^{\mathrm{b}} \pm 1.564$ & $23.72_{\mathrm{a}}^{\mathrm{a}} \pm 1.276$ & $21.16^{\mathrm{b}} \pm 2.031$ & $44.25_{\mathrm{b}}^{\mathrm{a}} \pm 4.327$ & $44.44_{b}{ }^{a} \pm 3.071$ \\
\hline \multicolumn{11}{|c|}{ Semitendinosus (ST) } \\
\hline 4 & $44.06_{a}^{a} \pm 3.166$ & $42.28_{a}{ }^{a} \pm 2.814$ & $.06_{\mathrm{b}}{ }^{\mathrm{a}} \pm 1.434$ & $13.07_{b}^{b} \pm 1.655$ & $15.25^{\mathrm{a}} \pm 1.107$ & $13.16_{\mathrm{c}}^{\mathrm{b}} \pm 1.321$ & $21.46_{b}{ }^{a} \pm 1.612$ & $18.64_{c}^{b} \pm 1.488$ & $45.46_{\mathrm{a}}^{\mathrm{a}} \pm 2.172$ & $45.47_{\mathrm{bc}}^{\mathrm{a}} \pm 4.541$ \\
\hline 7 & $44.82^{a}{ }^{a} \pm 4.364$ & $44.07 \mathrm{a}^{\mathrm{a}} \pm 4.205$ & $15.11_{\mathrm{b}}^{\mathrm{a}} \pm 1.569$ & $12.80^{b}+1.511$ & $16.09_{a b c}{ }^{a} \pm 1.560$ & $15.14_{\mathrm{ab}}^{\mathrm{a}} \pm 2.298$ & $22.16^{a b}{ }^{a} \pm 1.117$ & $19.91_{b c}^{b} \pm 2.133$ & $46.87^{\mathrm{a}} \mathrm{a} \pm 4.935$ & $49.60_{\mathrm{a}}^{\mathrm{a}} \pm 4.865$ \\
\hline 10 & $41.24^{a}{ }^{a} \pm 1.759$ & $42.15^{a} \pm 1.917$ & $16.74^{a} \pm 1.530$ & $13.75_{a b}{ }^{b} \pm 2.335$ & $16.80_{\mathrm{ab}}^{\mathrm{a}} \pm 1.252$ & $15.47_{\mathrm{a}}^{\mathrm{a}} \pm 1.777$ & $23.78_{a}^{a} \pm 0.905$ & $20.78_{a b}^{b} \pm 2.311$ & $45.17_{\mathrm{a}}^{\mathrm{b}} \pm 4.323$ & $48.59^{\mathrm{a}} \pm 4.984$ \\
\hline 13 & $43.34_{a b}{ }^{a} \pm 2.814$ & $42.80^{a}{ }^{a} \pm 2.745$ & $16.79^{a}{ }^{a} \pm 1.319$ & $14.83_{a}{ }^{b} \pm 1.681$ & $16.58_{\mathrm{abc}}{ }^{\mathrm{a}} \pm 1.849$ & $16.08^{a}{ }^{a} \pm 2.064$ & $23.65_{a}^{a} \pm 1.775$ & $21.90_{a}^{b} \pm 2.509$ & $44.68_{a b}^{a} \pm 3.501$ & $47.36_{a b}{ }^{a} \pm 2.227$ \\
\hline 16 & $45.35_{a}{ }^{a} \pm 3.366$ & $43.99^{a}{ }^{a} \pm 3.024$ & $16.01_{\mathrm{ab}^{\mathrm{a}}}^{\mathrm{a}} \pm 1.675$ & $14.42^{\mathrm{b}} \pm 1.671$ & $17.22^{a}{ }^{a} \pm 0.464$ & $15.93_{\mathrm{a}}^{\mathrm{a}} \pm 0.968$ & $23.55_{a}{ }^{a} \pm 1.268$ & $21.55^{b} \pm 1.264$ & $47.22_{a}{ }^{a} \pm 2.901$ & $48.05_{\mathrm{ab}}^{\mathrm{a}} \pm 3.987$ \\
\hline 19 & $43.56_{a b}{ }^{a} \pm 3.893$ & $43.62^{a} \pm 3.970$ & $17.28^{a} a^{a} \pm 1.874$ & $14.022_{a b} b^{b} \pm 1.095$ & 15.37 са \pm 1.077 & $13.63_{\mathrm{bc}}^{\mathrm{b}} \pm 1.873$ & $23.19^{a}{ }^{a} \pm 1.497$ & $19.61_{\mathrm{bc}}^{\mathrm{b}} \pm 1.556$ & $41.83^{a}{ }^{a} \pm 3.833$ & $44.09_{c}^{\mathrm{a}} \pm 4.487$ \\
\hline
\end{tabular}


Table 4 (continued) Means $( \pm \text { s.d.) })^{\#}$ for colour attributes of the raw beef muscles infused with a phosphate and lactate blend and aged for 19 days

\begin{tabular}{|c|c|c|c|c|c|c|c|c|c|c|}
\hline \multirow{2}{*}{ Day } & \multicolumn{2}{|c|}{ Raw L* } & \multicolumn{2}{|c|}{ Raw a* } & \multicolumn{2}{|c|}{ Raw b* } & \multicolumn{2}{|c|}{ Raw chroma } & \multicolumn{2}{|c|}{ Raw hue angle } \\
\hline & Control & Infused & Control & Infused & Control & Infused & Control & Infused & Control & Infused \\
\hline \multicolumn{11}{|c|}{ Supraspinatus (SS) } \\
\hline 4 & $39.99_{\mathrm{a}}^{\mathrm{a}} \pm 0.989$ & $38.93_{\mathrm{a}}{ }^{\mathrm{a}} \pm 1.928$ & $14.76_{\mathrm{b}}{ }^{\mathrm{a}} \pm 0.556$ & $13.74_{b}{ }^{a} \pm 1.365$ & $12.88_{b}{ }^{a} \pm 0.811$ & $12.09_{\mathrm{ab}}{ }^{\mathrm{a}} \pm 0.843$ & $19.65_{b}{ }^{a} \pm 0.793$ & $18.42_{\mathrm{b}}{ }^{\mathrm{a}} \pm 1.403$ & $40.97_{\mathrm{a}}^{\mathrm{a}} \pm 1.895$ & $41.40_{\mathrm{a}}{ }^{\mathrm{a}} \pm 2.233$ \\
\hline 7 & $40.17_{a}^{a} \pm 3.023$ & $37.09_{a}^{b} \pm 2.607$ & $15.87^{\mathrm{a}} \pm 1.062$ & $13.93_{b}^{b} \pm 1.206$ & $12.92_{\mathrm{b}}^{\mathrm{a}} \pm 1.076$ & $11.53_{b}{ }^{a} \pm 0.852$ & $20.51_{b}^{a} \pm 0.875$ & $18.14^{b}{ }^{b} \pm 1.233$ & $39.11_{a}{ }^{a} \pm 3.446$ & $39.66_{a}^{a} \pm 2.610$ \\
\hline 10 & $40.67 \mathrm{a}^{\mathrm{a}} \pm 1.377$ & $38.61_{\mathrm{a}}{ }^{\mathrm{a}} \pm 1.803$ & $18.26_{a}{ }^{a} \pm 0.993$ & $15.87_{\mathrm{a}}^{\mathrm{b}} \pm 0.938$ & $15.83^{a}{ }^{a} \pm 1.048$ & $12.58_{a b}{ }^{b} \pm 1.439$ & $24.18^{a}{ }^{a} \pm 1.352$ & $20.27 a^{b} \pm 1.508$ & $40.90_{\mathrm{a}}^{\mathrm{a}} \pm 1.184$ & $38.32_{a}^{a} \pm 2.216$ \\
\hline 13 & $40.73_{\mathrm{a}}{ }^{\mathrm{a}} \pm 2.051$ & $39.14^{a}{ }^{a} \pm 3.873$ & $18.77_{\mathrm{a}}^{\mathrm{a}} \pm 1.753$ & $15.74^{\mathrm{b}} \pm 1.631$ & $15.88_{a}{ }^{a} \pm 1.400$ & $13.60_{\mathrm{a}}^{\mathrm{b}} \pm 2.657$ & $24.59_{\mathrm{a}}^{\mathrm{a}} \pm 2.204$ & $20.84_{a}^{b} \pm 2.888$ & $40.24^{a} \pm 0.979$ & $40.49_{a}^{a} \pm 3.130$ \\
\hline 16 & $41.13^{a}{ }^{a} \pm 2.218$ & $38.58^{b} \pm 2.092$ & $17.83_{\mathrm{a}}{ }^{\mathrm{a}} \pm 0.677$ & $15.68^{\mathrm{b}} \pm 0.973$ & $14.95_{\mathrm{a}}^{\mathrm{a}} \pm 1.067$ & $13.53^{a}{ }^{a} \pm 1.256$ & $23.34_{\mathrm{a}}^{\mathrm{a}} \pm 0.928$ & $20.74^{b} \pm 1.403$ & $39.80_{\mathrm{a}}^{\mathrm{a}} \pm 2.166$ & $40.70_{\mathrm{a}}{ }^{\mathrm{a}} \pm 2.016$ \\
\hline 19 & $42.28_{a}{ }^{a} \pm 1.687$ & $38.77_{a}^{b} \pm 1.631$ & $17.84_{a}{ }^{a} \pm 0.561$ & $14.79_{\mathrm{ab}}^{\mathrm{b}} \pm 0.848$ & $15.68_{a}{ }^{a} \pm 0.730$ & $12.94_{a b}{ }^{b} \pm 1.448$ & $23.79_{a}{ }^{a} \pm 0.594$ & $19.69_{\mathrm{ab}}^{\mathrm{b}} \pm 1.485$ & $41.32_{\mathrm{a}}^{\mathrm{a}} \pm 4.686$ & $41.06_{a}{ }^{a} \pm 2.147$ \\
\hline \multicolumn{11}{|c|}{ Longissimus lumborum (LL) } \\
\hline 4 & $38.72_{c}{ }^{a} \pm 1.417$ & $38.16_{b}{ }^{a} \pm 1.723$ & $13.82_{\mathrm{c}}^{\mathrm{a}} \pm 1.048$ & $13.58_{\mathrm{cd}}{ }^{\mathrm{b}} \pm 1.004$ & $12.32_{c}{ }^{a} \pm 0.900$ & $12.08_{b}{ }^{a} \pm 0.775$ & $18.57_{c}^{a} \pm 1.103$ & $18.23_{\mathrm{c}}{ }^{\mathrm{a}} \pm 0.770$ & $41.62_{\mathrm{ab}}^{\mathrm{a}} \pm 2.409$ & $41.67_{a b}{ }^{a} \pm 3.150$ \\
\hline 7 & $40.56_{a b c}{ }^{a} \pm 1.725$ & $39.42_{\mathrm{ab}}{ }^{\mathrm{a}} \pm 1.141$ & $16.54_{b}{ }^{a} \pm 0.906$ & $14.36_{\mathrm{bcd}}^{\mathrm{b}} \pm 1.205$ & $14.15_{\mathrm{b}}^{\mathrm{a}} \pm 1.415$ & $12.73_{\mathrm{ab}}{ }^{\mathrm{a}} \pm 1.633$ & $21.87_{\mathrm{b}}^{\mathrm{a}} \pm 1.342$ & $19.23_{\mathrm{bc}}^{\mathrm{b}} \pm 1.904$ & $40.56_{\mathrm{ab}}^{\mathrm{a}} \pm 2.101$ & $41.37_{\mathrm{ab}}^{\mathrm{a}} \pm 2.159$ \\
\hline 10 & $39.39_{\mathrm{bc}}{ }^{\mathrm{a}} \pm 2.107$ & $38.59_{\mathrm{ab}}{ }^{\mathrm{a}} \pm 1.899$ & $16.04_{b}{ }^{a} \pm 0.948$ & $13.44_{d}^{b} \pm 1.494$ & $14.80_{\mathrm{ab}}{ }^{\mathrm{a}} \pm 0.334$ & $13.09_{a b}{ }^{b} \pm 0.894$ & $21.86_{b}^{a} \pm 0.756$ & $18.89_{\mathrm{bc}}^{\mathrm{b}} \pm 1.022$ & $42.75_{a}^{a} \pm 1.711$ & $44.32_{\mathrm{a}}{ }^{\mathrm{a}} \pm 4.357$ \\
\hline 13 & $41.24_{a b}{ }^{a} \pm 2.026$ & $39.67_{a b}{ }^{a} \pm 2.186$ & $17.25_{\mathrm{ab}}{ }^{\mathrm{a}} \pm 0.887$ & $14.83_{\mathrm{bc}}^{\mathrm{b}} \pm 1.423$ & $15.87_{\mathrm{a}}^{\mathrm{a}} \pm 0.658$ & $13.93^{\mathrm{b}} \pm 0.341$ & $23.47_{\mathrm{ab}}{ }^{\mathrm{a}} \pm 0.891$ & $20.39_{a b}{ }^{b} \pm 1.224$ & $42.58_{a}{ }^{a} \pm 1.553$ & $43.29_{\mathrm{a}}^{\mathrm{a}} \pm 2.311$ \\
\hline 16 & $40.17_{\mathrm{abc}}^{\mathrm{a}} \pm 1.630$ & $39.62_{a b}{ }^{a} \pm 1.178$ & $16.31_{\mathrm{b}}{ }^{\mathrm{a}} \pm 1.370$ & $15.09_{b}{ }^{a} \pm 0.914$ & $14.55_{a b}{ }^{a} \pm 0.932$ & $13.34_{a b}^{a} \pm 0.767$ & $21.90_{\mathrm{b}}^{\mathrm{a}} \pm 1.265$ & $20.20_{\mathrm{ab}}^{\mathrm{b}} \pm 0.559$ & $41.74_{a b}{ }^{a} \pm 2.742$ & $41.49_{\mathrm{ab}}{ }^{\mathrm{a}} \pm 2.988$ \\
\hline 19 & $41.91_{\mathrm{a}}{ }^{\mathrm{a}} \pm 1.248$ & $40.74_{a}^{a} \pm 1.064$ & $18.38 \mathrm{a}^{\mathrm{a}} \pm 0.456$ & $16.79_{a}^{b} \pm 0.903$ & $15.02_{\mathrm{ab}}{ }^{\mathrm{a}} \pm 0.835$ & $13.81_{\mathrm{a}}^{\mathrm{a}} \pm 1.278$ & $23.76_{a}^{a} \pm 0.471$ & $21.76_{a}{ }^{b} \pm 1.333$ & $39.25_{\mathrm{b}}^{\mathrm{a}} \pm 1.896$ & $39.37_{b}^{a} \pm 2.205$ \\
\hline $\begin{array}{c}\text { LSD } \\
P=0.05 \\
\end{array}$ & \multicolumn{2}{|c|}{2.336} & \multicolumn{2}{|c|}{1.277} & \multicolumn{2}{|c|}{1.528} & \multicolumn{2}{|c|}{1.631} & \multicolumn{2}{|c|}{3.108} \\
\hline
\end{tabular}

\# s.d.: standard deviation.

a, b, c, d, e Column means between days within a treatment and within a muscle with common subscripts do not differ $(P \leq 0.05)$.

${ }^{a, b}$ Row means between treatments within an attribute with common superscripts do not differ $(P \leq 0.05)$.

LSD: Least significant difference $(P=0.05)$. 
Table 5 Summary of means ( \pm s.d.) $)^{\#}$ for colour attributes of the raw beef muscles infused with a phosphate and lactate blend and aged for 19 days

\begin{tabular}{|c|c|c|c|c|c|c|c|c|c|c|}
\hline \multirow{2}{*}{ Muscle } & \multicolumn{2}{|c|}{ Raw L* } & \multicolumn{2}{|c|}{ Raw a* } & \multicolumn{2}{|c|}{ Raw b* } & \multicolumn{2}{|c|}{ Raw chroma } & \multicolumn{2}{|c|}{ Raw hue angle } \\
\hline & Control & Infused & Control & Infused & Control & Infused & Control & Infused & Control & Infused \\
\hline $\mathrm{BF}$ & $40.0_{c}{ }^{a} \pm 1.982$ & $39.04_{b}^{b} \pm 2.124$ & $17.38^{a} \pm 2.005$ & $15.79^{b} \pm 1.772$ & $15.04_{\text {bc }}{ }^{a} \pm 2.053$ & $13.93_{\mathrm{b}}^{\mathrm{b}} \pm 1.703$ & $23.02^{a}{ }^{a} \pm 2.699$ & $21.11_{\mathrm{a}}^{\mathrm{b}} \pm 2.245$ & $40.75_{\mathrm{b}}^{\mathrm{a}} \pm 2.424$ & $41.33_{\mathrm{cd}} \mathrm{a} \pm 2.724$ \\
\hline $\mathrm{RF}$ & $45.0_{a}{ }^{a} \pm 3.677$ & $42.97^{\mathrm{b}} \pm 3.326$ & $15.31_{c}{ }^{a} \pm 1.809$ & $13.67_{c}^{b} \pm 1.958$ & $15.61_{\mathrm{ab}}{ }^{\mathrm{a}} \pm 1.400$ & $13.59_{\mathrm{bc}}^{\mathrm{b}} \pm 1.830$ & $21.94_{b}^{a}{ }^{a} \pm 1.789$ & $19.36^{\mathrm{b}} \pm 2.346$ & $45.63_{a}^{a} \pm 3.693$ & $44.89_{b}{ }^{a} \pm 3.805$ \\
\hline ST & $43.8_{\mathrm{b}}^{\mathrm{a}} \pm 3.367$ & $43.15_{a}{ }^{a} \pm 3.18$ & $16.16_{\mathrm{b}}^{\mathrm{a}} \pm 1.694$ & $13.81_{c}{ }^{b} \pm 1.728$ & $16.22_{\mathrm{a}}^{\mathrm{a}} \pm 1.404$ & $14.90_{\mathrm{a}}^{\mathrm{b}} \pm 1.995$ & $22.96_{a}^{a} \pm 1.558$ & $20.40_{\mathrm{b}}^{\mathrm{b}} \pm 2.127$ & $45.20_{a}^{b} \pm 3.881$ & $47.19_{a}^{a} \pm 4.398$ \\
\hline SS & $40.83_{c}^{a} \pm 2.001$ & $38.52_{b}^{b} \pm 2.361$ & $17.22_{\mathrm{a}}^{\mathrm{a}} \pm 1.724$ & $14.96_{b}^{b} \pm 1.412$ & $14.69_{c}^{a} \pm 1.637$ & $12.71_{d}^{b} \pm 1.612$ & $22.68_{a}^{a} \pm 2.236$ & $19.68_{\mathrm{c}}^{\mathrm{b}} \pm 1.942$ & $40.39_{\mathrm{b}}^{\mathrm{a}} \pm 2.054$ & $40.27_{d}^{\mathrm{a}} \pm 2.471$ \\
\hline LL & $40.33_{c}{ }^{\mathrm{a}} \pm 1.928$ & $39.36^{b}{ }^{b} \pm 1.694$ & $16.39_{\mathrm{b}}^{\mathrm{a}} \pm 1.666$ & $14.68^{b}{ }^{b} \pm 1.575$ & $14.45_{c}{ }^{\mathrm{a}} \pm 1.386$ & $13.16_{\mathrm{cd}}^{\mathrm{b}} \pm 1.151$ & $21.91_{\mathrm{b}}^{\mathrm{a}} \pm 1.950$ & $19.78_{b c}{ }^{b} \pm 1.624$ & $41.41_{b}{ }^{a} \pm 2.313$ & $41.92_{c}{ }^{a} \pm 3.169$ \\
\hline $\begin{array}{c}\text { LSD } \\
P=0.05\end{array}$ & \multicolumn{2}{|c|}{0.953} & \multicolumn{2}{|c|}{0.521} & \multicolumn{2}{|c|}{0.624} & \multicolumn{2}{|c|}{0.666} & \multicolumn{2}{|c|}{1.269} \\
\hline
\end{tabular}

\# s.d.: standard deviation.

BF: biceps femoris; RF: rectus femoris; ST: semitendinosus; SS: supraspinatus; LL: longissimus lumborum.

a, b, c, d Column means within a treatment and between muscles with common subscripts do not differ $(P \leq 0.05)$.

${ }_{a, b}^{a, b}$ Row means within an attribute and between treatments with common superscripts do not differ $(P \leq 0.05)$.

LSD: least significant difference $(P=0.05)$. 
Table 6 Means ( \pm s.d.) $)^{\#}$ for colour attributes of the cooked beef muscles infused with a phosphate and lactate blend and aged for 19 days

\begin{tabular}{|c|c|c|c|c|c|c|c|c|c|c|}
\hline \multirow{2}{*}{ Day } & \multicolumn{2}{|c|}{ Cooked L* } & \multicolumn{2}{|c|}{ Cooked $a^{*}$} & \multicolumn{2}{|c|}{ Cooked b* } & \multicolumn{2}{|c|}{ Cooked chroma } & \multicolumn{2}{|c|}{ Cooked hue angle } \\
\hline & Control & Infused & Control & Infused & Control & Infused & Control & Infused & Control & Infused \\
\hline \multicolumn{11}{|c|}{ Biceps femoris (BF) } \\
\hline 4 & $38.88_{a b}{ }^{a} \pm 2.800$ & $42.00_{a}{ }^{a} \pm 2.129$ & $5.74_{b c}^{a} \pm 0.896$ & $5.72_{a}^{a} \pm 0.501$ & $13.11_{c}{ }^{a} \pm 0.785$ & $12.55_{c}^{\mathrm{a}} \pm 0.417$ & $14.34_{d}{ }^{a} \pm 0.629$ & $13.82_{c}{ }^{a} \pm 0.439$ & $66.30_{\mathrm{bc}}{ }^{\mathrm{a}} \pm 3.998$ & $65.44_{\mathrm{b}}^{\mathrm{a}} \pm 1.911$ \\
\hline 7 & $39.67_{\mathrm{ab}}{ }^{\mathrm{a}} \pm 3.208$ & $40.92^{a}{ }^{a} \pm 2.515$ & $5.91_{b c}^{a} \pm 1.186$ & $6.18^{a}{ }^{a} \pm 0.703$ & $15.35^{a}{ }^{a} \pm 0.805$ & $14.69_{\mathrm{ab}}^{\mathrm{a}} \pm 0.610$ & $16.50_{b c}{ }^{a} \pm 0.889$ & $15.96^{a}{ }^{a} \pm 0.627$ & $68.96_{\mathrm{ab}}^{\mathrm{a}} \pm 3.896$ & $67.14_{a b}^{a} \pm 2.458$ \\
\hline 10 & $41.36_{a}^{a} \pm 1.374$ & $42.51_{a}^{a} \pm 3.431$ & $5.48_{c}^{a} \pm 0.711$ & $5.25_{a}^{a} \pm 0.538$ & $15.87_{a}^{a} \pm 0.338$ & $14.64_{\mathrm{ab}}^{\mathrm{b}} \pm 0.457$ & $16.82_{a b}{ }^{a} \pm 0.201$ & $15.58_{a b}^{b} \pm 0.470$ & $70.95_{a}^{a} \pm 2.608$ & $70.28^{a}{ }^{a} \pm 1.874$ \\
\hline 13 & $41.75_{a}^{a} \pm 4.417$ & $41.80^{a}{ }^{a} \pm 4.710$ & $5.92_{\mathrm{bc}}{ }^{\mathrm{a}} \pm 1.217$ & $5.83_{a}^{a} \pm 0.809$ & $15.99_{a}^{a} \pm 0.853$ & $14.98^{\mathrm{b}} \pm 0.891$ & $17.10_{a b}{ }^{a} \pm 0.794$ & $16.10^{\mathrm{b}} \pm 0.837$ & $69.67^{a} a^{a} \pm 4.221$ & $68.70_{a b}^{a} \pm 3.049$ \\
\hline 19 & $41.48^{a} \pm 1.580$ & $41.80_{\mathrm{a}}^{\mathrm{a}} \pm 1.824$ & $6.51_{\mathrm{ab}}^{\mathrm{a}} \pm 0.847$ & $5.76_{a}^{a} \pm 0.685$ & $15.97 \mathrm{a}^{\mathrm{a}} \pm 0.793$ & $15.23_{\mathrm{a}}^{\mathrm{a}} \pm 0.728$ & $17.27_{\mathrm{a}}^{\mathrm{a}} \pm 0.993$ & $16.29^{b} \pm 0.801$ & $67.85_{\mathrm{ab}}^{\mathrm{a}} \pm 1.975$ & $69.27_{a}^{a} \pm 2.117$ \\
\hline \multicolumn{11}{|c|}{ Rectus femoris (RF) } \\
\hline 4 & $50.52_{a}^{a} \pm 2.709$ & $50.78 \mathrm{a}^{\mathrm{a}} \pm 2.992$ & $4.15_{\mathrm{b}}^{\mathrm{a}} \pm 0.531$ & $3.99_{\mathrm{a}}^{\mathrm{a}} \pm 1.562$ & $16.47_{\mathrm{b}}^{\mathrm{a}} \pm 0.505$ & $16.65_{\mathrm{a}}^{\mathrm{a}} \pm 0.734$ & $17.01_{b}{ }^{a} \pm 0.536$ & $17.19 a^{a} \pm 0.878$ & $75.77_{\mathrm{ab}}^{\mathrm{a}} \pm 1.727$ & $76.58_{a}^{a} \pm 4.853$ \\
\hline 7 & $46.21_{\mathrm{b}}^{\mathrm{a}} \pm 4.447$ & $47.03_{b}{ }^{a} \pm 3.365$ & $4.87_{a b}^{a} \pm 0.984$ & $4.09_{a}^{a} \pm 0.576$ & $17.06_{\mathrm{ab}}^{\mathrm{a}} \pm 0.446$ & $15.72^{\mathrm{b}} \mathrm{b} \pm 0.769$ & $17.85_{a}^{a} \pm 0.391$ & $16.32_{b}^{b} \pm 0.631$ & $73.95_{\mathrm{abc}}{ }^{\mathrm{a}} \pm 3.324$ & $75.05_{a b}{ }^{a} \pm 2.473$ \\
\hline 10 & $45.82_{b}{ }^{a} \pm 3.681$ & $45.46_{b}{ }^{a} \pm 3.368$ & $5.12_{\mathrm{a}}^{\mathrm{a}} \pm 0.801$ & $4.31_{\mathrm{a}}^{\mathrm{a}} \pm 0.920$ & $16.69_{b}{ }^{a} \pm 0.398$ & $15.75_{b}^{b} \pm 0.663$ & $17.56_{a b}{ }^{a} \pm 0.250$ & $16.39_{b}^{b} \pm 0.533$ & $72.72_{\mathrm{bc}}{ }^{\mathrm{a}} \pm 3.008$ & $74.53_{a b}{ }^{a} \pm 3.536$ \\
\hline 13 & $45.21_{b}{ }^{a} \pm 3.854$ & $44.84_{b}{ }^{a} \pm 3.427$ & $5.22_{\mathrm{a}}^{\mathrm{a}} \pm 0.548$ & $4.74_{a}{ }^{a} \pm 0.818$ & $16.90_{\mathrm{ab}}^{\mathrm{a}} \pm 0.868$ & $16.22_{a b}{ }^{a} \pm 0.959$ & $17.80_{\mathrm{a}}{ }^{\mathrm{a}} \pm 0.652$ & $16.99_{a b}^{b} \pm 0.722$ & $72.33_{c}{ }^{a} \pm 2.875$ & $73.38_{a b}^{a} \pm 3.579$ \\
\hline 16 & $45.62_{\mathrm{b}}^{\mathrm{a}} \pm 2.936$ & $47.53^{a}{ }^{a} \pm 3.174$ & $5.18_{\mathrm{a}}^{\mathrm{a}} \pm 1.054$ & $4.00_{a}^{b} \pm 0.888$ & $16.67_{\mathrm{b}}^{\mathrm{a}} \pm 0.741$ & $16.24_{\mathrm{ab}}{ }^{\mathrm{a}} \pm 0.685$ & $17.50_{\mathrm{ab}}{ }^{\mathrm{a}} \pm 0.490$ & $16.79_{\mathrm{ab}}^{\mathrm{a}} \pm 0.527$ & $72.64_{b c}^{b} \pm 3.898$ & $76.07_{a b}^{a} \pm 3.436$ \\
\hline \multicolumn{11}{|c|}{ Semitendinosus (ST) } \\
\hline 4 & $42.42_{a}^{a} \pm 3.653$ & $45.34_{a}^{a} \pm 4.337$ & $7.06_{a}^{a} \pm 0.764$ & $6.54_{a}{ }^{a} \pm 1.242$ & $15.67_{c}^{b} \pm 0.773$ & $16.96_{a}^{a} \pm 0.585$ & $17.23_{\mathrm{ab}}^{\mathrm{b}} \pm 0.504$ & $18.23_{\mathrm{a}}^{\mathrm{a}} \pm 0.475$ & $65.72_{\mathrm{c}}^{\mathrm{a}} \pm 3.246$ & $68.90_{\mathrm{b}}^{\mathrm{a}} \pm 4.081$ \\
\hline 7 & $42.11_{\mathrm{a}}^{\mathrm{a}} \pm 4.049$ & $44.70_{a}{ }^{a} \pm 3.410$ & $6.16_{\mathrm{ab}}^{\mathrm{a}} \pm 1.032$ & $5.35_{\mathrm{b}}{ }^{\mathrm{a}} \pm 1.235$ & $15.76_{\mathrm{bc}}^{\mathrm{a}} \pm 0.945$ & $15.86_{\mathrm{bc}}^{\mathrm{a}} \pm 0.998$ & $16.97_{b}^{a} \pm 0.573$ & $16.81_{b c}{ }^{a} \pm 0.829$ & $68.56_{\mathrm{bc}}^{\mathrm{a}} \pm 4.411$ & $71.25_{\mathrm{ab}}^{\mathrm{a}} \pm 4.586$ \\
\hline 10 & $42.34_{a}^{b} \pm 4.46$ & $46.39 a^{a} \pm 4.948$ & $5.12_{c}^{\mathrm{a}} \pm 0.887$ & $4.70_{b}{ }^{a} \pm 1.158$ & $16.13_{\mathrm{abc}}^{\mathrm{a}} \pm 0.931$ & $16.21_{\mathrm{abc}}{ }^{\mathrm{a}} \pm 0.595$ & $16.98_{b}^{a} \pm 0.707$ & $16.93_{b c}{ }^{a} \pm 0.565$ & $72.24_{a}^{a} \pm 3.658$ & $73.87_{\mathrm{a}}^{\mathrm{a}} \pm 4.004$ \\
\hline 13 & $43.35^{a}{ }^{a} \pm 4.59$ & $46.19^{a}{ }^{a} \pm 2.608$ & $5.76_{\mathrm{bc}}{ }^{\mathrm{a}} \pm 1.264$ & $5.15_{b}{ }^{a} \pm 0.634$ & $16.68^{a}{ }^{a} \pm 0.930$ & $16.46_{a b}{ }^{a} \pm 0.618$ & $17.71_{a}^{a} \pm 0.629$ & $17.28_{b}{ }^{a} \pm 0.718$ & $70.91_{\mathrm{ab}}^{\mathrm{a}} \pm 4.464$ & $72.74_{a}^{a} \pm 1.735$ \\
\hline 16 & $43.13_{a}{ }^{a} \pm 5.54$ & $45.79_{a}{ }^{a} \pm 4.481$ & $5.44_{b c}{ }^{a} \pm 1.357$ & $4.75_{b}{ }^{a} \pm 0.775$ & $15.91_{\mathrm{abc}}{ }^{\mathrm{a}} \pm 0.897$ & $15.54_{c}^{\mathrm{a}} \pm 0.632$ & $16.89_{b}{ }^{a} \pm 0.521$ & $16.29_{c}{ }^{a} \pm 0.493$ & $71.01_{\mathrm{ab}}^{\mathrm{a}} \pm 5.332$ & $72.97{ }_{\mathrm{a}}^{\mathrm{a}} \pm 3.092$ \\
\hline 19 & $45.25^{a}{ }^{a} \pm 4.698$ & $47.10^{a}{ }^{a} \pm 2.316$ & $5.26_{\mathrm{bc}}{ }^{\mathrm{a}} \pm 1.019$ & $5.11_{b}{ }^{a} \pm 0.334$ & $16.52_{a b}{ }^{a} \pm 0.808$ & $15.62_{c}^{\mathrm{b}} \pm 0.658$ & $17.38_{a b}{ }^{a} \pm 0.641$ & $16.46^{b} \pm 0.562$ & $72.24^{a}{ }^{a} \pm 3.756$ & $71.87 \mathrm{ab}^{\mathrm{a}} \pm 1.656$ \\
\hline
\end{tabular}


Table 6 (continued) Means ( \pm s.d.) $)^{\#}$ for colour attributes of the cooked beef muscles infused with a phosphate and lactate blend and aged for 19 days

\begin{tabular}{|c|c|c|c|c|c|c|c|c|c|c|}
\hline \multirow{2}{*}{ Day } & \multicolumn{2}{|c|}{ Cooked L* } & \multicolumn{2}{|c|}{ Cooked a* } & \multicolumn{2}{|c|}{ Cooked b* } & \multicolumn{2}{|c|}{ Cooked chroma } & \multicolumn{2}{|c|}{ Cooked hue angle } \\
\hline & Control & Infused & Control & Infused & Control & Infused & Control & Infused & Control & Infused \\
\hline \multicolumn{11}{|c|}{ Supraspinatus (SS) } \\
\hline 4 & $39.87^{a} \pm 2.658$ & $39.90_{a}{ }^{a} \pm 3.997$ & $6.90_{\mathrm{a}}^{\mathrm{a}} \pm 0.754$ & $6.27_{\mathrm{a}}^{\mathrm{a}} \pm 0.690$ & $15.30_{\mathrm{b}}{ }^{\mathrm{a}} \pm 0.525$ & $14.76{ }_{a b c}{ }^{a} \pm 0.998$ & $16.82_{b}{ }^{a} \pm 0.639$ & $16.08^{b} \pm 0.882$ & $65.69_{\mathrm{b}}^{\mathrm{a}} \pm 2.121$ & $66.95_{\mathrm{b}}^{\mathrm{a}} \pm 3.037$ \\
\hline 7 & $38.50_{a}^{a} \pm 3.002$ & $38.47_{a}^{a} \pm 2.632$ & $7.04^{a}{ }^{a} \pm 0.965$ & $6.23^{a}{ }^{a} \pm 0.403$ & $15.92_{a b}{ }^{a} \pm 0.682$ & $14.58^{b} \pm 0.940$ & $17.45_{\mathrm{ab}}{ }^{\mathrm{a}} \pm 0.532$ & $15.90^{\mathrm{b}} \pm 0.956$ & $66.13^{\mathrm{a}}{ }^{\mathrm{a}} \pm 3.478$ & $66.74_{b}{ }^{a} \pm 1.316$ \\
\hline 10 & $39.32^{a}{ }^{a} \pm 2.388$ & $38.81_{a}{ }^{a} \pm 2.827$ & $7.09_{a}^{a} \pm 0.725$ & $6.38_{a}^{a} \pm 0.610$ & $15.96_{a b}{ }^{a} \pm 0.898$ & $14.65_{\mathrm{bc}}^{\mathrm{b}} \pm 0.769$ & $17.55_{\mathrm{a}}{ }^{\mathrm{a}} \pm 0.642$ & $16.02^{\mathrm{b}} \pm 0.532$ & $65.83_{\mathrm{b}}^{\mathrm{a}} \pm 3.275$ & $66.36_{b}{ }^{a} \pm 2.908$ \\
\hline 13 & $38.86_{a}^{a} \pm 3.452$ & $39.42^{a}{ }^{a} \pm 2.790$ & $5.93_{b}{ }^{a} \pm 0.931$ & $5.11_{b}{ }^{a} \pm 0.678$ & $16.22_{a}{ }^{a} \pm 0.806$ & $15.48_{a}^{a} \pm 0.968$ & $17.32_{a b}{ }^{a} \pm 0.495$ & $16.34^{\mathrm{b}} \pm 0.716$ & $69.80_{\mathrm{a}}^{\mathrm{a}} \pm 3.802$ & $71.57 a^{a} \pm 3.278$ \\
\hline 16 & $37.36_{a}^{a} \pm 2.029$ & $39.00_{a}^{a} \pm 2.367$ & $6.67_{a b}^{a} \pm 0.673$ & $5.98_{a b}{ }^{a} \pm 0.652$ & $16.30^{a}{ }^{a} \pm 0.516$ & $15.42_{a b}{ }^{b} \pm 0.629$ & $17.65^{a}{ }^{a} \pm 0.578$ & $16.59^{\mathrm{b}} \pm 0.449$ & $67.74_{a b}^{a} \pm 2.099$ & $68.75_{a b}^{a} \pm 2.761$ \\
\hline 19 & $39.04_{a}^{a} \pm 3.757$ & $40.29^{a}{ }^{a} \pm 2.353$ & $6.69_{\mathrm{ab}}{ }^{\mathrm{a}} \pm 0.701$ & $6.31_{a}^{a} \pm 0.665$ & $16.11_{a}^{a}{ }^{a} \pm 1.044$ & $15.30_{a b c}{ }^{b} \pm 0.796$ & $17.49_{\mathrm{ab}}^{\mathrm{a}} \pm 0.911$ & $16.59_{a}^{\mathrm{b}} \pm 0.695$ & $67.38_{\mathrm{ab}}^{\mathrm{a}} \pm 2.814$ & $67.55_{b}{ }^{a} \pm 2.670$ \\
\hline \multicolumn{11}{|c|}{ Longissimus lumborum $(L L)$} \\
\hline 4 & $49.39_{a}^{a} \pm 3.395$ & $48.19_{\mathrm{a}}^{\mathrm{a}} \pm 4.910$ & $5.33_{\mathrm{a}}^{\mathrm{a}} \pm 1.296$ & $4.37_{\mathrm{a}}^{\mathrm{b}} \pm 1.124$ & $17.56_{\mathrm{a}}{ }^{\mathrm{a}} \pm 1.235$ & $15.29^{b} \pm 0.792$ & $18.42_{a}{ }^{a} \pm 1.382$ & $15.94^{\mathrm{b}} \pm 0.779$ & $73.31_{\mathrm{ab}}{ }^{\mathrm{a}} \pm 3.482$ & $74.07_{c}^{a} \pm 4.082$ \\
\hline 7 & $46.97_{a b}{ }^{a} \pm 4.089$ & $49.31_{a}^{a} \pm 2.596$ & $5.41_{a}^{a} \pm 0.797$ & $4.22_{a b}^{b} \pm 0.692$ & $15.81_{b}{ }^{a} \pm 0.504$ & $15.59^{a}{ }^{a} \pm 0.363$ & $16.74_{c}{ }^{a} \pm 0.510$ & $16.17_{a}^{a} \pm 0.406$ & $71.08_{b}^{b} \pm 2.776$ & $74.90_{\mathrm{bc}}{ }^{\mathrm{a}} \pm 2.367$ \\
\hline 10 & $48.27_{a b}{ }^{a} \pm 3.973$ & $51.06_{a}^{a} \pm 4.337$ & $4.34_{b}^{a} \pm 1.204$ & $3.41_{b c}{ }^{b} \pm 1.584$ & $16.99_{a}^{a} \pm 0.542$ & $15.54^{\mathrm{b}} \pm 0.359$ & $17.59_{b}{ }^{a} \pm 0.330$ & $15.99^{\mathrm{b}}{ }^{\mathrm{b}} \pm 0.325$ & $75.62^{a} \pm 4.176$ & $77.62_{\mathrm{ab}}{ }^{\mathrm{a}} \pm 5.739$ \\
\hline 13 & $49.62_{\mathrm{a}}^{\mathrm{a}} \pm 4.821$ & $50.70_{\mathrm{a}}{ }^{\mathrm{a}} \pm 3.901$ & $4.28_{\mathrm{b}}{ }^{\mathrm{a}} \pm 0.795$ & $3.74_{a b c}{ }^{a} \pm 0.997$ & $17.04_{a}^{a} \pm 0.068$ & $15.79_{a}^{b} \pm 0.691$ & $17.59_{b}{ }^{a} \pm 0.174$ & $16.26_{\mathrm{a}}^{\mathrm{b}} \pm 0.785$ & $75.92_{\mathrm{a}}^{\mathrm{a}} \pm 2.527$ & $76.70_{\mathrm{abc}}{ }^{\mathrm{a}} \pm 3.340$ \\
\hline 16 & $45.42^{\mathrm{b}}{ }^{\mathrm{b}} \pm 4.012$ & $49.57_{a}^{a} \pm 2.193$ & $4.21_{\mathrm{b}}{ }^{\mathrm{a}} \pm 0.645$ & $3.15^{b} \pm 0.524$ & $16.77^{a}{ }^{a} \pm 0.724$ & $15.80^{a} a^{b} \pm 0.426$ & $17.31_{\mathrm{bc}}{ }^{\mathrm{a}} \pm 0.543$ & $16.13^{\mathrm{a}}{ }^{\mathrm{b}} \pm 0.485$ & $75.83^{a}{ }^{a} \pm 2.708$ & $78.69_{a}^{a} \pm 1.728$ \\
\hline 19 & $46.74_{\mathrm{ab}}^{\mathrm{b}} \pm 3.275$ & $50.07_{a}^{a} \pm 2.738$ & $4.30_{\mathrm{b}}{ }^{\mathrm{a}} \pm 0.895$ & $3.17_{c}^{b} \pm 0.618$ & $16.82_{a}^{a} \pm 0.651$ & $15.54_{a}^{\mathrm{b}} \pm 0.398$ & $17.40_{b c}{ }^{a} \pm 0.636$ & $15.90_{\mathrm{a}}^{\mathrm{b}} \pm 0.430$ & $75.61_{a}^{a} \pm 3.035$ & $78.39_{a}{ }^{a} \pm 2.236$ \\
\hline $\begin{array}{c}\text { LSD } \\
P=0.05\end{array}$ & \multicolumn{2}{|c|}{3.178} & \multicolumn{2}{|c|}{0.930} & \multicolumn{2}{|c|}{0.795} & \multicolumn{2}{|c|}{0.722} & \multicolumn{2}{|c|}{3.350} \\
\hline
\end{tabular}

s.d.: standard deviation.

a, b, c, d Column means between days within a treatment and within a muscle with common subscripts do not differ $(P \leq 0.05)$.

${ }^{a, b}$ Row means between treatments within an attribute with common superscripts do not differ $(P \leq 0.05)$.

LSD: least significant difference $(P=0.05)$. 
Table 7 Summary of means ( \pm s.d.) $)^{\#}$ for colour attributes of the cooked beef muscles infused with a phosphate and lactate blend and aged for 19 days

\begin{tabular}{|c|c|c|c|c|c|c|c|c|c|c|}
\hline \multirow{2}{*}{ Muscle } & \multicolumn{2}{|c|}{ Cooked L* } & \multicolumn{2}{|c|}{ Cooked a* } & \multicolumn{2}{|c|}{ Cooked b* } & \multicolumn{2}{|c|}{ Cooked chroma } & \multicolumn{2}{|c|}{ Cooked hue angle } \\
\hline & Control & Infused & Control & Infused & Control & Infused & Control & Infused & Control & Infused \\
\hline $\mathrm{BF}$ & $40.00_{c}^{b} \pm 3.298$ & $41.60_{c}{ }^{a} \pm 3.060$ & $6.07_{\mathrm{b}}^{\mathrm{a}} \pm 1.018$ & $5.72_{a}^{a} \pm 0.666$ & $15.11_{\mathrm{c}}^{\mathrm{a}} \pm 1.306$ & $14.37_{\mathrm{d}}^{\mathrm{b}} \pm 1.097$ & $16.33_{\mathrm{c}}^{\mathrm{a}} \pm 1.233$ & $15.49^{\mathrm{b}} \pm 1.043$ & $68.02_{c}{ }^{a} \pm 3.905$ & $68.18_{d}{ }^{a} \pm 2.757$ \\
\hline $\mathrm{RF}$ & $47.18_{\mathrm{a}}^{\mathrm{a}} \pm 4.040$ & $47.10_{\mathrm{b}}{ }^{\mathrm{a}} \pm 3.585$ & $4.75_{c}^{a} \pm 0.974$ & $4.33_{c}{ }^{b} \pm 0.943$ & $16.89_{a}^{a} \pm 0.671$ & $16.07_{\mathrm{a}}^{\mathrm{b}} \pm 0.750$ & $17.62^{a} \pm 0.575$ & $16.71_{\mathrm{a}}^{\mathrm{b}} \pm 0.670$ & $74.10_{\mathrm{a}}^{\mathrm{a}} \pm 3.490$ & $74.75_{\mathrm{b}}{ }^{\mathrm{a}} \pm 3.434$ \\
\hline $\mathrm{ST}$ & $43.10_{b}^{b} \pm 4.334$ & $45.92_{b}{ }^{a} \pm 3.614$ & $5.80_{\mathrm{b}}^{\mathrm{a}} \pm 1.197$ & $5.27_{\mathrm{b}}^{\mathrm{b}} \pm 1.084$ & $16.11_{\mathrm{b}}{ }^{\mathrm{a}} \pm 0.888$ & $16.11_{\mathrm{a}}^{\mathrm{a}} \pm 0.819$ & $17.19_{\mathrm{b}}^{\mathrm{a}} \pm 0.627$ & $17.00_{\mathrm{a}}{ }^{\mathrm{a}} \pm 0.864$ & $70.12_{b}^{b} \pm 4.543$ & $71.93_{c}{ }^{a} \pm 3.530$ \\
\hline SS & $38.83_{c}^{a} \pm 2.836$ & $39.31_{d}^{a} \pm 2.743$ & $6.72_{a}^{a} \pm 0.837$ & $6.05^{\mathrm{b}} \pm 0.728$ & $15.97_{\mathrm{b}}^{\mathrm{a}} \pm 0.790$ & $15.03_{c}^{b} \pm 0.883$ & $17.83_{a b}{ }^{a} \pm 0.659$ & $16.25_{b}^{b} \pm 0.728$ & $67.09_{c}{ }^{a} \pm 3.138$ & $67.99_{d}{ }^{a} \pm 3.108$ \\
\hline LL & $47.73_{\mathrm{a}}^{\mathrm{b}} \pm 3.970$ & $49.82^{a}{ }^{a} \pm 3.456$ & $4.64_{c}^{\mathrm{a}} \pm 1.037$ & $3.67_{d}^{b} \pm 1.039$ & $16.83_{a}^{a} \pm 0.845$ & $15.59_{\mathrm{b}}^{\mathrm{b}} \pm 0.524$ & $17.51_{a}{ }^{a} \pm 0.826$ & $16.07_{b}^{b} \pm 0.539$ & $74.56^{\mathrm{b}} \pm 3.452$ & $76.73_{a}{ }^{a} \pm 3.698$ \\
\hline $\begin{array}{c}\text { LSD } \\
P=0.05\end{array}$ & \multicolumn{2}{|c|}{1.2975} & \multicolumn{2}{|c|}{0.3797} & \multicolumn{2}{|c|}{0.3246} & \multicolumn{2}{|c|}{0.2947} & \multicolumn{2}{|c|}{1.3677} \\
\hline
\end{tabular}

BF: biceps femoris; RF: rectus femoris; ST: semitendinosus; SS: supraspinatus; LL: longissimus lumborum.

a, b, c, d Column means within a treatment and between muscles with common subscripts do not differ $(P \leq 0.05)$.

a, ${ }^{2}$ Row means within an attribute and between treatments with common superscripts do not differ $(P \leq 0.05)$. LSD: least significant difference $(P=0.05)$.

Table 8 Means $( \pm \text { s.d.) })^{\#}$ for proximate chemical composition of beef muscles infused with a phosphate and lactate blend

\begin{tabular}{|c|c|c|c|c|c|c|c|c|}
\hline \multirow{2}{*}{ Muscle } & \multicolumn{2}{|c|}{ Moisture (\%) } & \multicolumn{2}{|c|}{ Protein (\%) } & \multicolumn{2}{|c|}{ Lipid (\%) } & \multicolumn{2}{|c|}{ Ash (\%) } \\
\hline & Control & Infused & Control & Infused & Control & Infused & Control & Infused \\
\hline $\mathrm{BF}$ & $73.17_{\mathrm{b}}^{\mathrm{a}} \pm 1.548$ & $73.61_{c}^{a} \pm 1.489$ & $20.21_{\mathrm{a}}^{\mathrm{a}} \pm 1.203$ & $18.13_{\mathrm{bc}}^{\mathrm{b}} \pm 0.814$ & $3.04^{\mathrm{b}} \pm 0.971$ & $3.71_{a}^{a} \pm 0.726$ & $1.13_{\mathrm{ab}}^{\mathrm{b}} \pm 0.081$ & $1.73_{\mathrm{b}}{ }^{\mathrm{a}} \pm 0.121$ \\
\hline $\mathrm{RF}$ & $74.18_{a b}^{b} \pm 0.747$ & $75.84_{\mathrm{ab}}{ }^{\mathrm{a}} \pm 1.325$ & $20.28_{\mathrm{a}}^{\mathrm{a}} \pm 0.955$ & $17.19_{c}^{b} \pm 2.140$ & $2.54_{\mathrm{ab}}^{\mathrm{a}} \pm 0.523$ & $2.54_{b}{ }^{a} \pm 0.783$ & $1.15_{\mathrm{ab}}^{\mathrm{b}} \pm 0.020$ & $1.91_{\mathrm{a}}^{\mathrm{a}} \pm 0.087$ \\
\hline ST & $75.10_{\mathrm{a}}^{\mathrm{b}} \pm 0.936$ & $76.99_{a}^{a} \pm 1.250$ & $20.71_{\mathrm{a}}^{\mathrm{a}} \pm 0.800$ & $19.25_{\mathrm{ab}}^{\mathrm{a}} \pm 1.214$ & $2.10_{\mathrm{b}}^{\mathrm{a}} \pm 0.435$ & $1.70_{c}{ }^{a} \pm 0.261$ & $1.14_{a b}^{b} \pm 0.094$ & $1.89_{\mathrm{a}}^{\mathrm{a}} \pm 0.094$ \\
\hline SS & $75.22_{\mathrm{a}}^{\mathrm{b}} \pm 0.931$ & $76.84^{a}{ }^{a} \pm 1.191$ & $20.28_{\mathrm{a}}^{\mathrm{a}} \pm 0.942$ & $18.82_{\mathrm{abc}}{ }^{\mathrm{a}} \pm 1.043$ & $2.95_{a}^{a} \pm 0.617$ & $2.47_{\mathrm{b}}^{\mathrm{a}} \pm 0.378$ & $1.06_{\mathrm{b}}^{\mathrm{b}} \pm 0.144$ & $1.74_{b}{ }^{a} \pm 0.122$ \\
\hline LL & $73.84_{b}{ }^{a} \pm 0.685$ & $74.62_{b c}{ }^{a} \pm 1.160$ & $19.78_{\mathrm{a}}^{\mathrm{a}} \pm 2.538$ & $20.16_{a}^{a} \pm 1.190$ & $2.28_{\mathrm{b}}{ }^{\mathrm{a}} \pm 0.335$ & $2.51_{b}{ }^{a} \pm 0.780$ & $1.25_{\mathrm{a}}^{\mathrm{b}} \pm 0.098$ & $1.72_{b}{ }^{a} \pm 0.106$ \\
\hline $\begin{array}{c}\text { LSD } \\
P=0.05\end{array}$ & \multicolumn{2}{|c|}{1.260} & \multicolumn{2}{|c|}{1.681} & \multicolumn{2}{|c|}{0.582} & \multicolumn{2}{|c|}{0.116} \\
\hline
\end{tabular}

\# s.d.: Standard deviation.

BF: biceps femoris; RF: rectus femoris; ST: semitendinosus; SS: supraspinatus ; LL: longissimus lumborum.

a, b, c Column means within a treatment and between muscles with common subscripts do not $(P \leq 0.05)$.

${ }_{\mathrm{a}, \mathrm{b}}^{\mathrm{a}}$ Row means within a component and between treatments with common superscripts do not differ $(P \leq 0.05)$. LSD: least significant difference $(P=0.05)$. 
Table 9 Means $( \pm \text { s.d.) })^{\#}$ for mineral composition (mg/100 g) of beef muscles infused with a phosphate and lactate blend

\begin{tabular}{|c|c|c|c|c|c|c|c|c|c|c|c|}
\hline \multirow{3}{*}{$\begin{array}{l}\text { Mineral component } \\
\text { (mg/100 g) }\end{array}$} & \multicolumn{10}{|c|}{ Muscle } & \multirow{3}{*}{$\begin{array}{c}\text { LSD } \\
(P=0.05)\end{array}$} \\
\hline & \multicolumn{2}{|c|}{$\begin{array}{c}\text { Biceps femoris } \\
(B F)\end{array}$} & \multicolumn{2}{|c|}{$\begin{array}{l}\text { Rectus femoris } \\
(R F)\end{array}$} & \multicolumn{2}{|c|}{$\begin{array}{l}\text { Semitendinosus } \\
(S T)\end{array}$} & \multicolumn{2}{|c|}{$\begin{array}{c}\text { Supraspinatus } \\
\text { (SS) }\end{array}$} & \multicolumn{2}{|c|}{$\begin{array}{c}\text { Longissimus lumborum } \\
(L L)\end{array}$} & \\
\hline & Control & Infused & Control & Infused & Control & Infused & Control & Infused & Control & Infused & \\
\hline Phosphorus & $180.0_{\mathrm{ab}}{ }^{\mathrm{a}} \pm 9.928$ & $157.9_{\mathrm{b}}{ }^{\mathrm{a}} \pm 29.78$ & $196.3^{a} \pm 8.138$ & $178.5_{\mathrm{ab}}{ }^{\mathrm{a}} \pm 25.04$ & $164.1_{\mathrm{bc}}{ }^{\mathrm{a}} \pm 5.198$ & $184.0_{a}{ }^{a} \pm 35.53$ & $154.6_{c}{ }^{a} \pm 9.572$ & $172.8_{a b}{ }^{a} \pm 14.57$ & $189.8^{a}{ }^{a} \pm 7.922$ & $158.8_{b}{ }^{b} \pm 25.42$ & 23.58 \\
\hline Potassium & $166.8_{a b}{ }^{b} \pm 16.28$ & $191.7_{\mathrm{b}}^{\mathrm{a}} \pm 28.93$ & $163.6_{a b}{ }^{b} \pm 3.209$ & $199.7_{a b}{ }^{a} \pm 26.53$ & $161.5_{\mathrm{ab}}^{\mathrm{b}} \pm 7.329$ & $215.4_{a}{ }^{a} \pm 35.92$ & $145.5_{\mathrm{b}}^{\mathrm{b}} \pm 8.043$ & $196.6_{a b}{ }^{a} \pm 20.06$ & $169.9_{a}{ }^{a} \pm 5.086$ & $187.5^{a}{ }^{a} \pm 12.40$ & 22.56 \\
\hline Calcium & $6.35_{a}{ }^{a} \pm 1.301$ & $4.94^{b} \pm 1.167$ & $6.98^{a} \pm 0.199$ & $4.73_{\mathrm{b}}^{\mathrm{b}} \pm 0.889$ & $6.46_{a}{ }^{a} \pm 0.984$ & $5.64_{b}{ }^{a} \pm 0.878$ & $6.89_{a}^{a} \pm 0.622$ & $7.89_{a}^{a} \pm 1.813$ & $7.17_{\mathrm{a}}^{\mathrm{a}} \pm 0.164$ & $4.94_{b}^{b} \pm 0.809$ & 1.126 \\
\hline Magnesium & $21.78^{a} \pm 0.795$ & $16.29_{\mathrm{ab}}^{\mathrm{b}} \pm 1.130$ & $22.49^{a} \pm 1.126$ & $15.35_{\mathrm{b}}^{\mathrm{b}} \pm 2.093$ & $22.80_{a}{ }^{a} \pm 0.845$ & $16.34_{\mathrm{ab}}^{\mathrm{b}} \pm 1.491$ & $21.85_{a}^{a} \pm 1.885$ & $17.56_{a}^{b} \pm 0.922$ & $21.88_{a}{ }^{a} \pm 1.706$ & $16.41_{\mathrm{ab}}^{\mathrm{b}} \pm 1.943$ & 1.895 \\
\hline Sodium & $12.05^{\mathrm{b}} \pm 1.442$ & $24.43_{b c}{ }^{a} \pm 5.879$ & $11.15_{a}{ }^{b} \pm 0.454$ & $25.74_{b}{ }^{\mathrm{a}} \pm 4.298$ & $10.91_{\mathrm{a}}^{\mathrm{b}} \pm 0.316$ & $26.27_{\mathrm{b}}^{\mathrm{a}} \pm 3.537$ & $12.59_{\mathrm{a}}^{\mathrm{b}} \pm 0.974$ & $30.99_{a}^{a} \pm 5.358$ & $11.49_{\mathrm{a}}^{\mathrm{b}} \pm 0.569$ & $21.30_{c}{ }^{a} \pm 3.108$ & 3.968 \\
\hline Iron & $2.73^{a} \pm 0.608$ & $1.99_{\mathrm{b}}^{\mathrm{b}} \pm 0.561$ & $1.91_{\mathrm{b}}^{\mathrm{a}} \pm 0.390$ & $1.73_{\mathrm{b}}^{\mathrm{a}} \pm 0.346$ & $2.11_{b}^{\mathrm{a}} \pm 0.326$ & $1.58_{b}^{b} \pm 0.232$ & $2.95_{a}^{a} \pm 0.350$ & $2.68{ }_{a}^{a} \pm 0.368$ & $2.15_{b}{ }^{a} \pm 0.323$ & $1.63_{b}{ }^{b} \pm 0.201$ & 0.454 \\
\hline Copper & $0.018_{\mathrm{ab}}{ }^{\mathrm{a}} \pm 0.008$ & $0.025_{\mathrm{ab}}{ }^{\mathrm{a}} \pm 0.005$ & $0.022_{\mathrm{a}}^{\mathrm{a}} \pm 0.004$ & $0.023_{\mathrm{b}}{ }^{\mathrm{a}} \pm 0.005$ & $0.017_{\mathrm{ab}}^{\mathrm{b}} \pm 0.005$ & $0.032_{\mathrm{a}}^{\mathrm{a}} \pm 0.004$ & $0.013_{\mathrm{b}}^{\mathrm{b}} \pm 0.008$ & $0.027_{\mathrm{ab}}{ }^{\mathrm{a}} \pm 0.008$ & $0.016_{a b}{ }^{a} \pm 0.005$ & $0.022_{\mathrm{b}}^{\mathrm{a}} \pm 0.008$ & 0.008 \\
\hline Zinc & $3.48^{a} a^{a} \pm 0.610$ & $2.28_{\mathrm{d}}^{\mathrm{b}} \pm 0.628$ & $4.49_{b}{ }^{a} \pm 0.554$ & $3.37_{b}^{b} \pm 0.187$ & $3.54_{c d}{ }^{a} \pm 0.637$ & $2.45_{\mathrm{cd}}^{\mathrm{b}} \pm 0.772$ & $6.03^{a} \pm 0.638$ & $4.74^{\mathrm{b}} \pm 0.433$ & $4.05_{b c}{ }^{a} \pm 0.627$ & $2.91_{b c}^{b} \pm 0.768^{f}$ & 0.540 \\
\hline Manganese & $0.028^{a}{ }^{a} \pm 0.004$ & $0.027_{\mathrm{a}}^{\mathrm{a}} \pm 0.005$ & $0.028_{a}{ }^{a} \pm 0.008$ & $0.020_{\mathrm{b}}^{\mathrm{b}} \pm 0.000$ & $0.010^{b}{ }^{b} \pm 0.000$ & $0.020_{\mathrm{b}}^{\mathrm{a}} \pm 0.009$ & $0.015_{b}{ }^{a} \pm 0.005$ & $0.015^{a}{ }^{a} \pm 0.005$ & $0.033^{a}{ }^{a} \pm 0.008$ & $0.030_{\mathrm{a}}^{\mathrm{a}} \pm 0.000$ & 0.006 \\
\hline
\end{tabular}

s.d.: standard deviation.

a, b, c, d Column means between days within a treatment and within a muscle with common subscripts do not differ $(P \leq 0.05)$.

Row means between treatments within an attribute with common superscripts do not differ $(P \leq 0.05)$.

LSD: least significant difference $(P=0.05)$. 
The ash content of the infused samples (Table 9) indicated an increase in mineral composition as a result of the blend. Therefore, significant differences in mineral content between treatments are expected. The infused muscle had higher concentrations for $\mathrm{K}, \mathrm{Na}$, and $\mathrm{Cu}$, which is expected, because both $\mathrm{K}$ and $\mathrm{Na}$ are present in the blend infused into the beef muscles. The levels of P were high only in the infused SS muscle - the reason is unknown as it would have been expected that the added phosphate would have accumulated within the infused muscle. The role of the $\mathrm{P}$ used in the phosphate blends needs further elucidation. The effect of the infusion on the various muscles is in accord with previous data (Hoffman, 2006).

\section{Conclusions}

One of the main objectives of the beef industry is to produce a product of consistent quality, which complies with consumer needs and satisfies the demand for a high-quality beef product (Kerth et al., 1995). In the present study the effect of a blend containing sodium and potassium salts, di- and triphosphates and lactates on the $\mathrm{pH}$, water-binding capacity, instrumental meat colour and instrumental tenderness during post-mortem ageing were investigated. The initial proximate and mineral composition of the treated muscles was also determined. The general findings suggest than an increase in tenderness concurrent with minimal changes in beef colour resulted from the infusion with a blend containing sodium and potassium salts, diand triphosphates and lactates. Thus, infusion with this blend is one of the methods that can be used by South African meat processors to improve traditionally less tender beef cuts. Several corrective actions that are referred to in this study have been investigated by researchers to overcome toughness problems, reduce tenderness variability and increase consumer satisfaction in beef quality (Scanga et al., 2000; Baublits et al., 2005a; b; Hoffman, 2006). The similarities of the brine solutions applied within these studies and the success achieved by other reported studies give an indication of the success that the blend used in the present investigation could accomplish in the South African beef industry. In conclusion, the infusion of beef muscles with a commercial basting mixture containing sodium and potassium salts, di- and triphosphates and lactates is an effective means of lowering shear force values, without negatively affecting the colour and water-binding abilities. Therefore, this blend could be implemented in current industry practice as a feasible and effective means of improving the tenderness of beef with no detrimental effects to other physical and chemical properties.

\section{References}

AOAC, 2002. Official Methods of Analysis, $16^{\text {th }}$ ed. Arlington, VA: Association of Official Analytical Chemists.

Baublits, R.T., Pohlman, F.W., Brown Jr., A.H. \& Johnson, Z.B., 2005a. Effects of sodium chloride, phosphate type and concentration, and pump rate on beef biceps femoris quality and sensory characteristics. Meat Sci. 70, 205-214.

Baublits, R.T., Pohlman, F.W., Brown Jr., A.H. \& Johnson, Z.B., 2005b. Effects of enhancement with varying phosphate types and concentrations, at two different pump rates on beef biceps femoris instrumental color characteristics. Meat Sci. 71, 264-276.

Baublits, R.T., Pohlman, F.W., Brown Jr., A.H. \& Johnson, Z.B., 2006. Impact of muscle type and sodium chloride concentration on the quality, sensory, and instrumental colour characteristics of solution enhanced whole-muscle beef. Meat Sci. 72, 704-712.

Belew, J.B., Brooks, J.C., McKenna, D.R. \& Savell, J.W., 2003. Warner-Bratzler shear evaluations of 40 bovine muscles. Meat Sci. 64, 507-512.

Bendall, J.R., 1967. The elastin content of various muscles of beef animals. J. Sci. Food Agric. 18, 553-558.

Benito-Delgado, J., Marriott, N.G., Claus, J.R., Wang, H. \& Graham, P.P., 1994. Chuck longissimus and infraspinatus muscle characteristics as affected by rigor state, blade tenderisation and calcium chloride injection. J. Food Sci. 59, 295-299.

Boleman, S.J., Boleman, S.L., Bidner, T.D., McMillin, K.W. \& Monlezun, C.J., 1995. Effects of postmortem time of calcium chloride injection on beef tenderness and drip, cooking and total loss. Meat Sci. 39, 35-41.

Boles, J.A. \& Shand, P.J., 2001. Meat cut and injection level affect the tenderness and cook yield of processed roast beef. Meat Sci. 59, 259-265. 
Bouton, P.E., Harris, P.V., Macfarlane, J.J. \& Shorthose, W.R., 1982. Influence of pH on the WarnerBratzler shear properties of mutton. Meat Sci. 6, 27-36.

Braghieri, A., Cifuni, G.F., Girolami, A., Riviezzi, A.M., Marsico, I. \& Napolitano, F., 2005. Chemical, physical and sensory properties of meat from pure and crossbred Podolian bulls at different ageing times. Meat Sci. 69, 681-689.

Briskey, E.J., Hoekstra, W.G., Bray, R.W. \& Grummer, R.H., 1960. A comparison of certain physical characteristics of eight pork muscles. J. Anim. Sci. 19, 214-225.

Carpenter, C.E., Cornforth, D.P. \& Whittier, D., 2001. Consumer preferences for beef colour and packaging did not affect eating satisfaction. Meat Sci. 57, 359-363.

Chen, C.M. \& Trout, G.R., 1991. Colour and its stability in restructured beef steaks during frozen storage: effects of various binders. J. Food Sci. 56, 1461-1464.

CIE., 1978. Recommendations on Uniform Colour Spaces - Colour Difference Equations Psychometric Colour Terms. Paris: Commission International de I'Eclairage Supplement No 2 to CIE Publication No 15 (E-1.3.1) 1971/(TC-1.3).

Crouse, J.D., Cross, H.R. \& Seideman, S.C., 1984. Effects of a grass or grain diet on the quality of three beef muscles. J. Anim. Sci. 58, 619-625.

Delgado, E.J., Rubio, M.S., Itube, F.A., Méndez, R.D., Cassís, L. \& Rosiles, R., 2005. Composition and quality of Mexican and imported retail beef in Mexico. Meat Sci. 69, 465-471.

Dransfield, E., Wakefield, D.K. \& Parkman, I.D., 1992. Modelling post-mortem tenderisation-I: Texture of electrically stimulated and non-stimulated beef. Meat Sci. 31, 57-73.

Duxbury, D.D., 1988. Natural sodium lactate extends shelf life of whole and ground meats. Food Proc. 49, 91.

French, P., O’Riordan, E.G., Monahan, F.J., Caffrey, P.J., Mooney, M.T., Troy, D.J. \& Moloney, A.P., 2001. The eating quality of meat of steers fed grass and/or concentrates. Meat Sci. 57, 379-386.

Giron, H.C., 1973. Dry ashing: method 6.1.1. Atomic Absorption Newsletter 12, 28.

Got, F., Culioli, J., Berge, P., Vignon, X., Astruc, T., Quideau, J.M. \& Lethiecq, M., 1999. Effects of high intensity high frequency ultrasound on ageing rate, ultrastructure and some physio-chemical properties of beef. Meat Sci. 51, 35-42.

Government Notice No R. 1748, 26 June, 1992. Regulation Gazette No 4890, Government Gazette No 14060. Volume 324, p. 8.

Greaser, M.L., 2001. Postmortem muscle chemistry. In: Meat Science and Applications. Eds Hui, Y.H., Nip, W.K., Rogers, R.W. \& Young, O.A., Chapter 2, Section 1. New York: Marcel Dekker, Inc. pp. 21-37

Grunert, K.G., 1997. What's in a steak? A cross-cultural study of the quality perception of beef. Food Qual. Pref. 8, 157-174.

Hamm, R., 1960. Biochemistry of meat hydration. Advances in Food Research, 10, 355-463. (As cited by Young et al., 1005).

Hamm, R., 1970. Interactions between phosphates and meat proteins. In: Symposium: Phosphates in Food Processing. Eds Deman, J.M. \& Melnychyn, P., Westport, CT: AVI Publishing, Inc. pp. 65-82.

Hoffman, L.C., 2006. Sensory and physical characteristics of enhanced vs. non-enhanced meat from mature cows. Meat Sci. 72, 195-202.

Hoffman, L.C., Muller, M.M. \& Vermaak A., 2008. Sensory and preference testing of selected beef muscles infused with a phosphate and lactate blend. Meat Sci. 80, 1055-1060.

Holmer, S.F., Kutzler, L.W., McKeith, F.K. \& Killefer, J., 2009. Sodium citrate as a replacement for sodium chloride in a brine solution when evaluated in cows of different backfat thickness. Meat Sci. 81, 349-356.

Honikel, K.O., 1987. The water binding of meat. Die Fleischwirtschaft 67, 1098-1102.

Honikel, K.O., 1998. Reference methods for the assessment of physical characteristics of meat. Meat Sci. 49, 447-457.

Huffman, K.L., Miller, M.F., Hoover, S.C., Wu, C.K., Brittin, H.C. \& Ramsey, C.B., 1996. Effect of beef tenderness on consumer satisfaction with steaks consumed in the home and restaurant. J. Anim. Sci. 74, 91-97.

Kerth, C.R., Miller, M.F. \& Ramsey, C.B., 1995. Improvement of beef tenderness and quality traits with calcium chloride injection in beef loins 48 hours post-mortem. J. Anim. Sci. 67, 934-942. 
Kim, Y.H., Hunt, M.C., Mancini, R.A., Seyfert, M., Loughin, T.M., Kropf, D.H., et al., 2006. Mechanism for lactate-color stabilization in injection-enhanced beef. J. Agric. Fd Chem. 54, 7856-7862.

Koohmaraie, M. \& Shackelford, S.D., 1991. Effect of calcium chloride infusion on the tenderness of lambs fed a $\beta$-adrenergic agonist. J. Anim. Sci, 69, 2463-2471.

Koohmaraie, M., Babiker, A.S., Schroeder, A.L., Merkel, R.A. \& Dutson, T.R., 1988. Acceleration of postmortem tenderisation in ovine carcasses through activation of $\mathrm{Ca}^{2+}$-dependent proteases. J. Food Sci. 53, 1638-1641.

Koohmaraie, M., Crouse, J.D. \& Mersmann, H.J., 1989. Acceleration of postmortem tenderisation in ovine carcasses through infusion of calcium chloride: effect of concentration and ionic strength. J. Anim. Sci. 67, 934-942.

Koohmaraie, M., Whipple, G. \& Crouse, J.D., 1990. Acceleration of post-mortem tenderisation in lamb and Brahman-cross carcasses through infusion of calcium chloride. J. Anim. Sci. 68, 1278-1283.

Lawrence, T.E., Dikeman, M.E., Hunt, M.C., Kastner, C.L. \& Johnson, D.E., 2003. Staged injection marination with calcium lactate, phosphate and salt may improve beef water-binding ability and palatability traits. Meat Sci. 65, 967-972.

Lawrence, T.E., Dikeman, M.E., Hunt, M.C., Kastner, C.L. \& Johnson, D.E., 2004. Effects of enhancing beef longissimus with phosphate plus salt, or calcium lactate plus non-phosphate water binders plus rosemary extract. Meat Sci. 67, 129-137.

Lawrie, R.A., 1998. Chemical and biochemical constitution of muscle. In: Lawrie’s Meat Science, $6^{\text {th }}$ ed., Cambridge: Woodhead Publishing, Ltd. pp. 58-95.

Lee, B.J., Hendricks, D.G. \& Cornforth, D.P., 1998. Effect of sodium phytate, sodium pyrophosphate and sodium tripolyphosphate on physico-chemical characteristics of restructured beef. Meat Sci. 50, 273-283.

Lee, C.M., Trevino, B. \& Chaiyawat, M., 1996. A simple and rapid solvent extraction method for determining total lipids in fish tissue. J. Assoc. Off. Anal. Chem. 79, 487-492.

Lennon, A.M., Moon, S.S., Ward, P., O’Neill, E.E. \& Kenny, T., 2006. Effects of enhancement procedures on whole and re-formed beef forequarter muscles. Meat Sci. 72, 513-517.

Maca, J.V., Miller, .R.K., Bigner, M.E., Lucia, L.M. \& Acuff, G.R., 1999. Sodium lactate and storage temperature effects on shelf-life of vacuum packaged beef top rounds. Meat Sci. 53, 23-29.

Mancini, R.A. \& Ramanathan, R., 2007. Sodium lactate influences myoglobin redox stability in vitro. Meat Sci. 78, 529-532.

Mandigo, R.W., 2002. Ingredient opportunities for case-ready meats. [Internet document]. URL http://www.foodproductdesign.com/archive/2002/0202DE.html. Accessed 25/02/2005.

Maria, G.A., Villaroel, M., Sanudo, C., Olleta, J.L. \& Gebresenbet, G., 2003. Effect of transport time and ageing on aspects of beef quality. Meat Sci. 65, 1335-1340.

McGee, M.R., Henry, K.L., Brooks, J.C., Ray, F.K. \& Morgan, J.B., 2003. Injection of sodium chloride, sodium tripolyphosphate, and sodium lactate improves the Warner-Bratzler shear and sensory characteristics of pre-cooked inside round roasts. Meat Sci. 64, 273-277.

Molina, M.E., Johnson, D.D., West, R.L. \& Gwartney, B.L., 2005. Enhancing palatability traits in beef chuck muscles. Meat Sci. 71, 52-61.

Morgan, J.B., Miller, R.K., Mendez, F.M., Hale, D.S. \& Savell, J.W., 1991a. Using calcium chloride injection to improve tenderness of beef from mature cows. J. Anim. Sci. 69, 4469-4476.

Morgan, J.B., Savell, J.W., Hale, D.S., Miller, R.K., Griffin, D.B., Cross, H.R. \& Shackelford, S.D., $1991 b$. National beef tenderness survey. J. Anim. Sci. 69, 3274-3283.

Morris, C.A., Theis, R.L., Miller, R.K., Acuff, G.R. \& Savell, J.W., 1997. Improving the flavour of calcium chloride and lactic acid injected mature beef top round steaks. Meat Sci. 45, 531-537.

O’Neill, D.J., Troy, D.J. \& Mullen, A.M., 2004. Determination of potential inherent variability when measuring beef quality. Meat Sci. 66, 765-770.

Ott, R.L., 1998. An Introduction to Statistical Methods and Data Analysis. Belmont, California: Duxbury Press. pp. 807-837.

Papadopoulos, L.S., Miller, R.K., Ringer, L.J. \& Cross, H.R., 1991. Sodium lactate effect on sensory characteristics, cooked meat colour and chemical composition. J. Food. Sci. 56, 621-626, 635.

Paterson, B.C., Parrish Jr., F.C. \& Stromer, M.H., 1988. Effects of salt and pyrophosphate on the physical and chemical properties of beef muscle. J. Food. Sci. 53, 1258-1265. 
Pietrasik, Z. \& Shand, P.J., 2011. Effects of moisture enhancement, enzyme treatment, and blade tenderization on the processing characteristics and tenderness of beef semimembranosus steaks. Meat Sci. 88, 8-13.

Polidori, P., Trabalza Marinucci, M., Fantuz, F., Renieri, C. \& Polidori, F., 2000. Tenderisation of wether lambs meat through pre-rigor infusion of calcium ions. Meat Sci. 55, 197-200.

Prestat, C., Jensen, J., McKeith, F.K. \& Brewer, M.S., 2002. Cooking method and endpoint temperature effects on sensory and colour characteristics of pumped pork loin chops. Meat Sci. 60, 395-400.

Puolanne, E.J., Ruusunen, M.H. \& Vainionpää, J.I., 2001. Combined effects of $\mathrm{NaCl}$ and raw meat pH on water-holding in cooked sausage with and without added phosphate. Meat Sci. 58, 1-7.

Rhee, M.S., Wheeler, T.L., Shackelford, S.D. \& Koohmaraie, M., 2004. Variation in palatability and biochemical traits within and among eleven beef muscles. J. Anim. Sci. 82, 534-550.

SAS, 2003. SAS/STAT User's Guide, Version 9, $1^{\text {st }}$ printing, Volume 3. SAS Institute Inc, SAS Campus Drive, Cary, North Carolina 27513, USA.

Sayed, N., Frans, Y. \& Schönfeldt, H.C., 1999. Composition of South African Foods. Milk and Milk Products, Eggs and Meat and Meat Products. Supplement to the MRC Food Composition Tables. Parow: Medical Research Council.

Scanga, J.A., Delmore, R.J., Ames, R.P., Belk, K.E., Tatum, J.D. \& Smith, G.C., 2000. Palatability of beef steaks marinated with solutions of calcium chloride, phosphate, and (or) beef-flavoring. Meat Sci. 55, 397-401.

Schönfeldt, H.C. \& Welgemoed, C., 1996. Composition of South African Beef. Pretoria, South Africa: South African Meat Board.

Shapiro, S.S. \& Wilk, M.B., 1965. An analysis of variance test for normality (complete samples). Biom. 52, 591-611.

Sheard, P.R., Nute, G.R., Richardson, R.I., Perry, A. \& Taylor, A.A., 1999. Injection of water and polyphosphate into pork to improve juiciness and tenderness after cooking. Meat Sci. 51, 371-376.

Sherman, P., 1962. The water-binding capacity of fresh pork. IV. The influence of ion absorption from neutral salts and polyphosphate on water retention of lean pork. Food Technol. 16, 91-95.

Shults, G.W., Russell, D.R. \& Wierbicki, E., 1972. Effect of condensed phosphates on pH, swelling and water-holding capacity of beef. J. Food Sci. 37, 860-863.

Simmons, N.J., Daly, C.C., Cummings, T.L., Morgan, S.K., Johnson, N.V. \& Lombard, A.C., 2008. Reassessing the principles of electrical stimulation. Meat Sci. 80, 110-122.

Smith, G.C., Savell, J.W., Clayton, R.P., Field, T.G., Griffin, D.B., Hale, D.S., Miller, M.F., Montgomery, T.H., Morgan, J.B., Tatum, J.D. \& Wise, J.W., 1992. The final report of the National Beef Quality Audit. Colorado, State University, Ft. Collins and Texas A\&M University: College Station. (As cited by Belew et al., 2003).

Solomon, M.B., Long, J.B. \& Eastridge, J.S., 1997. The Hydrodyne: a new process to improve beef tenderness. J. Anim. Sci. 75, 1534-1537.

Sørheim, O. \& Hildrum, K.I., 2002. Muscle stretching techniques for improving meat tenderness. Trends Food Sci. Technol. 13, 127-135.

Suman, S.P., Mancini, R.A., Ramanathan, R. \& Konda, M.R., 2009. Effect of lactate-enhancement, modified atmosphere packaging, and muscle source on the internal cooked colour of beef steaks. Meat Sci. 81, 644-670.

Stites, C.R., McKeith, F.K., Bechtel, P.J. \& Carr, T.R., 1989. Palatability and storage characteristics of precooked beef roasts. J. Food Sci. 54, 3-6.

Toohey, E.S., Van de Ven, R., Thompson, J.M., Geesink, G.H. \& Hopkins, D.L., 2012a. SmartStretch ${ }^{\mathrm{TM}}$ Technology. I. Improving the tenderness of sheep topsides ( $m$. semimembranosus) using a meat stretching device. Meat Sci. 91, 142-147.

Toohey, E.S., Van de Ven, R., Thompson, J.M., Geesink, G.H. \& Hopkins, D.L., 2012b. SmartStretch ${ }^{\mathrm{TM}}$ Technology. II. Improving the tenderness of leg meat from sheep using a meat stetching device. Meat Sci. 91, 125-130.

Trout, G.R. \& Schmidt, G.R., 1983. Utilization of phosphates in meat products. In: Proceedings of the $36^{\text {th }}$ Reciprocal Meat Conference. Fargo, ND. pp. 24-27. (As cited by Baublits et al., 2006). 
Trout, G.R. \& Schmidt, G.R., 1984. Effect of phosphate type and concentration, salt level and method of preparation on binding in restructured beef rolls. J. Food Sci. 49, 687-694.

Trout, G.R. \& Schmidt, G.R., 1986. Effect of phosphates on the functional properties of restructured beef rolls: the role of $\mathrm{pH}$, ionic strength, and phosphate type. J. Food Sci. 51, 1416-1423.

Voisey, P.W., 1976. Engineering assessment and critique of instruments used for meat tenderness evaluation. J. Text. Stud. 7, 11-48.

Vote, D.J., Platter, W.J., Tatum, J.D., Schmidt, G.R., Belk, K.E. \& Smith, G.C., 2000. Injection of beef strip loins with solutions containing sodium tripolyphosphate, sodium lactate and sodium chloride to enhance palatability. J. Anim. Sci. 78, 952-957.

Walsh, H., Martins, S., O’Neill, E.E., Kerry, J.P., Kenny, T. \& Ward, P., 2010. The effects of different cooking regimes on the cook yield and tenderness of non-injected and injection enhanced forequarter beef muscles. Meat Sci. 84, 444-448.

Wheeler. T.L., Koohmaraie, M. \& Crouse, J.D., 1991. Effects of calcium chloride injection and hot-boning on the tenderness of round muscles. J. Anim. Sci. 69, 4871-4875.

Wheeler. T.L., Koohmaraie, M., Lansdell, J.L., Siragusa, G.R. \& Miller, M.F., 1993. Effects of post-mortem injection time, injection level, and concentration of calcium chloride on beef quality traits. J. Anim. Sci. 71, 2965-2974.

Whipple, G.M., Koohmaraie, M., Dikeman, E. \& Crouse, J.D., 1990. Predicting beef longissimus tenderness from various biochemical and histological muscle traits. J. Anim. Sci. 68, 4193-4199.

Wulf, D.M. \& Wise, J.W., 1999. Measuring muscle colour on beef carcasses using the L*a*b* colour space. J. Anim. Sci. 77, 2418-2427.

Young, O.A., Priolo, A., Simmons, N.J. \& West, J., 1999. Effects of rigor attainment temperature on meat blooming and colour on display. Meat Sci. 52, 47-56.

Young, O.A., Zhang, S.X., Farouk, M.M. \& Podmore, C., 2005. Effects of pH adjustment with phosphates on attributes and functionalities of normal and high pH beef. Meat Sci. 70, 133-139. 